\title{
Multifractal analyses of response time series: A comparative study
}

\author{
Espen A. F. Ihlen
}

Published online: 23 March 2013

(C) Psychonomic Society, Inc. 2013

\begin{abstract}
Response time series with a non-Gaussian distribution and long-range dependent dynamics have been reported for several cognitive tasks. Conventional monofractal analyses numerically define a long-range dependency as a single scaling exponent, but they assume that the response times are Gaussian distributed. Ihlen and Vereijken (Journal of Experimental Psychology: General, 139, 436$463,2010)$ suggested multifractal extensions of the conventional monofractal analyses that are more suitable when the response time has a non-Gaussian distribution. Multifractal analyses estimate a multifractal spectrum of scaling exponents that contain the single exponent estimated by the conventional monofractal analyses. However, a comparison of the performance of multifractal analyses with behavioral variables has not yet been addressed. The present study compares the performance of seven multifractal analyses. The multifractal analyses were tested on multiplicative cascading noise that generates time series with a predefined multifractal spectrum and with a structure of variation that mimics intermittent response time variation. Time series with 1,024 and 4,096 samples were generated with additive noise and multiharmonic trends of two different magnitudes (signal-to-noise/trend ratio; 0.33 and 1). The results indicate that all multifractal analysis has individual pros and cons related to sample size, multifractality, and the presence of additive noise and trends in the response time series. The summary of pros and cons of the seven multifractal analyses provides a guideline for the choice of multifractal analyses of response time series and other behavioral variables.
\end{abstract}

E. A. F. Ihlen $(\bowtie)$

Department of Neuroscience, Norwegian University of Science and Technology, 7489 Trondheim, Norway

e-mail: espen.ihlen@ntnu.no
Keywords Multifractal $\cdot$ Multiplicative interaction · Wavelet $\cdot$ Scaling laws $\cdot$ Response series $\cdot$ Behavior . Variability

\section{Introduction}

Analyses of response time series have given insight into cognitive processes and mental organization in a wide variety of tasks such as simple reaction, word naming, choice decision, visual search, memory search, and lexical decision. Contemporary response time analyses have been developed within two paradigms: analyses of the response time distribution and analyses of the trial dependency of response time dynamics. Response time distributions are typically unimodal and positively skewed toward long response latencies. Consequently, distributions like the ex-Gaussian (Burbeck \& Luce, 1982), shifted Wald (Schwarz, 2001), lognormal (Ulrich \& Miller, 1993), Gamma (Van Zandt \& Ratcliff, 1995), Weibull (Logan, 1992), and power law (Holden, Van Orden, \& Turvey, 2009; Moscoso del Prado Martin 2009) distributions have been suggested as models to parameterize the response time distribution. However, all models assume that the response times are trial-independent random variables. In contrast, several studies conducted through the last decades have found that response times possess long-range trial dependency (Gilden, 2001; Gilden, Thornton, \& Mallon, 1995; Kello, Beltz, Holden, \& Van Orden, 2007; Kello et al., 2010; Van Orden, Holden, \& Turvey, 2003). The long-range trial dependency of response times is numerically defined by a scaling exponent obtained by monofractal analyses such as detrended fluctuation analysis, scaled window variance analysis, or rescaled range analysis, to mention but a few (cf. Delignières et al., 2006). All these analyses numerically define long-range trial 
dependency of response time series by a single exponent that is estimated by the scaling of the root-mean square, variance, or range. This scaling exponent is found to decrease with increased difficulty and an increased level of external perturbation of the response time task (cf. Diniz et al., 2011) and has been suggested to reflect the self-organization of cognitive processes (e.g., Van Orden et al., 2003).

The conventional numerical definition of a single scaling exponent assumes that response times are Gaussian distributed such that the response time variation is described by the secondorder statistical moment (i.e., variance) alone. However, response time distributions are found to be unimodal and rightskewed toward long response latencies across a range of cognitive tasks (Luce, 1986). The presence of a non-Gaussian response time distribution has two fundamental implications for the analyses of long-range trial dependency of the response time series. First, the presence of a non-Gaussian response time distribution indicates that the response time variation cannot be exclusively described by the scaling of variance alone but that the scaling of higher order statistical moments such as skewness and kurtosis has to be considered. Thus, the single scaling exponent defined by conventional monofractal analyses will define only the fraction of the trial dependency related to the variance of the response time series. Second, the presence of a non-Gaussian response time distribution indicates intermittent changes in the magnitude of response time variation. These intermittent changes might be due to modulation of the task difficulty, sensory feedback, or changes in participants' intention to act according to the task instruction. Furthermore, intermittent changes in variability have been shown to be a central feature for phase transition in a bimanual coordination task (Kelso, 1995) and other complex systems (Schaffer et al., 2009). Intermittent changes in the response time variation will provide temporal modulation of both the width and shape of the response time distribution and, consequently, temporal modulation of the scaling exponent.

Ihlen and Vereijken (2010) introduced multifractal analyses that are able to numerically define the scaling exponents of higher order statistical moments and the temporal modulation of a local scaling exponent. They suggested that the multifractal structure of response time series reflected phase transitions in the mental organization driven by multiplicative interactions across the temporal scales of the response time dynamics. Later, multifractal analyses of response times revealed temporal changes in the trial-dependent structure of the response series by modulation in task difficulty and performance feedback (Kuznetsov \& Wallot, 2011) and the presence of strong anticipation (Stephen \& Dixon, 2011). The presence of multifractal variation is also a central feature of healthy regulation of physiological processes like heart rate dynamics (Ivanov et al., 1999), and the absence of multifractal variation is related to several pathological conditions (Wang, Huang, Xie, Wang, \& Hu, 2007). However, none of the studies within behavioral research have compared the performance of the multifractal analyses a priori with their employment for behavioral variables.

The aim of the present study is to compare the performance of seven multifractal analyses and, consequently, to provide a guideline for the choice of multifractal analyses of response time series and other behavioral variables. The article describes and compares multifractal analyses and assumes that the reader is familiar with basic statistical concepts of fractal analyses. Readers unfamiliar with the basic concepts of fractal analyses are referred to Ihlen and Vereijken (2010) and Ihlen (2012) for an introduction to the topic.

\section{Method}

Multifractal analyses of a response time series are all conducted by a step-wise procedure illustrated in Fig. 1. First, the response time series is decomposed into a scale-dependent measure $\mu_{s, t}$ (see the top panel in Fig. 1). Second, the multifractal spectrum is computed either directly from $\mu_{s, t}$ (right panels along B arrows in Fig. 1) or indirectly by the $q$-order statistics of $\mu_{s, t}$ (left panels along A arrows in Fig. 1). The scale-dependent measure $\mu_{s, t}$ and $q$-order statistics differentiate between the different types of multifractal analyses and, consequently, between their potential pros and cons when applied to response time series. The seven multifractal analyses compared in the present study are introduced in the next three subsections according to their scaledependent measure $\mu_{s, t}$ and their $q$-order statistics.

\section{Scale-dependent measure}

Response time series are decomposed into both time and scale domains by (1) computing a scale-dependent measure $\mu_{s, t_{0}}$ in a floating trial interval $\left[t_{0}-s / 2, t_{0}+s / 2\right]$ centered at trial $t_{0}$ with sample size $s$ (i.e., scale) or by (2) computing a scale-dependent measure $\mu_{s, v}$ in the nonoverlapping trial interval $[(v-1) s+1$, $v s]$ with interval number $v$ and sample size $s$. The analyses that estimate the multifractal spectrum directly from $\mu_{s, t}$ use method 1 , whereas the analyses based on $q$-order statistics of $\mu_{s, t}$ use method 2. The scale-dependent measure of the seven multifractal analyses compared in the present study is defined below.

\section{Multifractal detrended fluctuation analysis}

Multifractal detrended fluctuation analysis (MFDFA; Kantelhardt et al., 2002) defines the scale-dependent measure $\mu_{s, v}$ as a root-mean square fluctuation of the integrated profile $y_{t}=\sum_{i=1}^{t}\left(x_{i}-\bar{x}\right)$ of the response time series $x_{i}$ around a scale-dependent trend $\widehat{y}_{s, t}$ within a nonoverlapping time interval $[(v-1) s+1, v s]$ : 


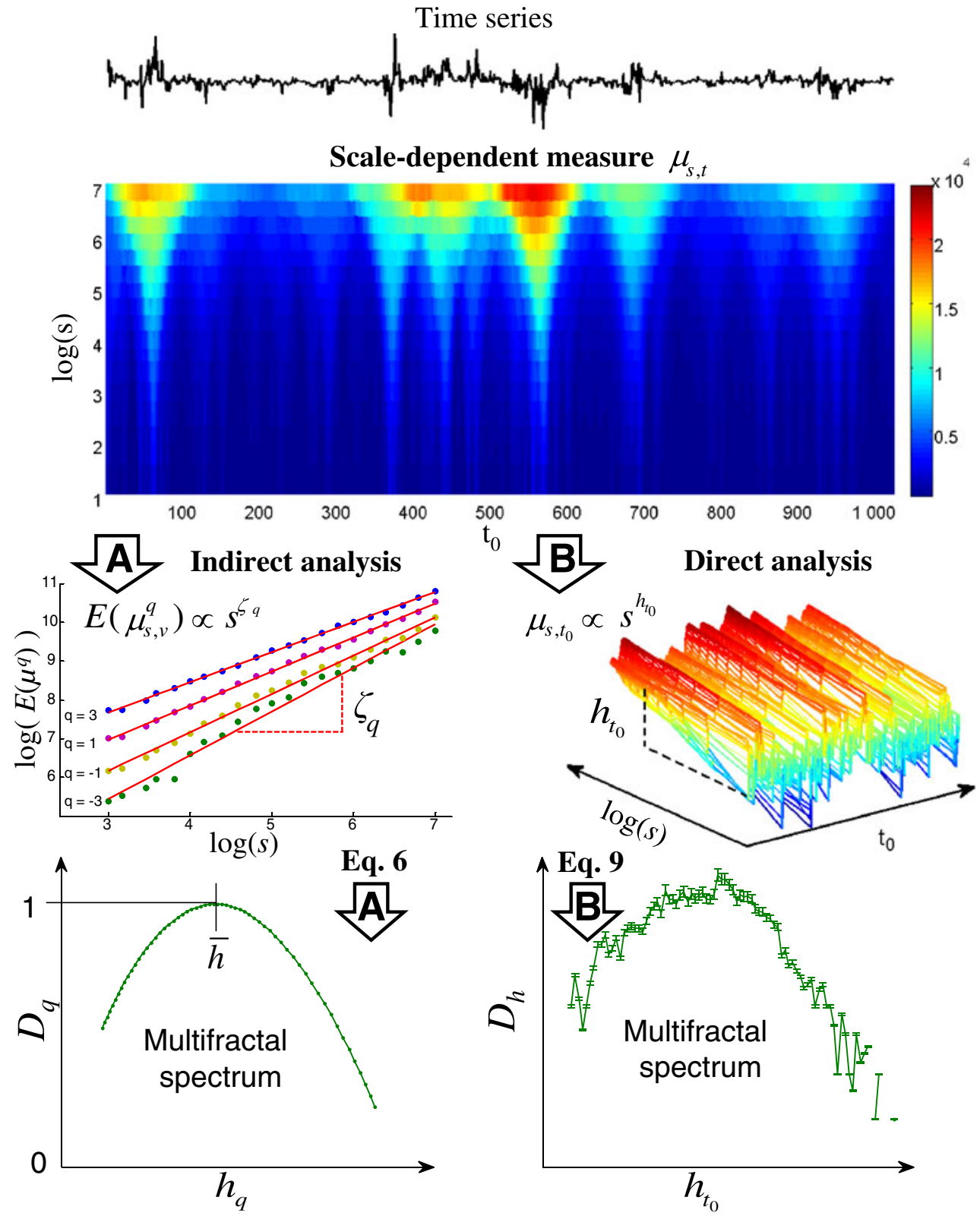

Fig. 1 A flow chart of the estimation of the multifractal spectrum $D_{h}$ directly from the scale-dependent measure $\mu_{s, t_{0}}$ (B arrows) and indirectly by the $q$-order statistics of $\mu_{s, v}$ (A arrows). The basis for all multifractal analyses within both formalisms is the scale-dependent measure (upper contour plot) that decomposes the response time series or behavioral variable into both the time and scale domains. The red contours indicate large scale-dependent measures of the response time series that coincide with the time periods of intermittent large variations. In contrast, the blue contours indicate small scale-dependent measures that coincide with the time periods of intermittent small variations. The panel below the top arrow A indicates that the scale-dependent measure is summarized by its $q$-order statistical moment (see Eq. 5). The statistical moments with

positive $q$ s amplifies the large $\mu_{s, v}$ (i.e., red contours), whereas the statistical moments negative $q$ s amplifies the small $\mu_{s, v}$ (i.e., blue contours). The scaling exponent $\zeta_{q}$ numerical defines the power law relation of the intermittent periods with large (i.e., positive $q$ s) and small variations (i.e., negative $q$ s). The panel below the bottom arrow A illustrates a multifractal spectrum $D_{h}$ estimated by the Legendre transformation of $\zeta_{q}$ (i.e., Eq. 6). The multifractal spectrum $D_{h}$ is the plot of $D_{q}$ versus $h_{q}$ where the mode $\bar{h}=h_{q=0}$ is defined at the maxima $D_{q=0}=1$. The panel below the top arrow B illustrates the direct estimation of the local singularity exponent $h_{t_{0}}$ as the local slope of $\log \left(\mu_{s, t_{0}}\right)$ versus $\log (s)$ for each time instant $t_{0}$. The panel below the bottom arrow B illustrates the multifractal spectrum $D_{h}$ defined by Eq. 9 in the main text

The present study compares the performance of MFDFA $\mu_{s, v}=\sqrt{\frac{1}{s} \sum_{t=(v-1) s+1}^{v s}\left[y_{t}-\hat{y}_{t}\right]^{2}}$ with linear $\left(\mathrm{MFDFA}_{\text {lin }}\right)$, moving average $\left(\mathrm{MFDFA}_{\mathrm{ma}}\right)$, and empirical mode decomposition $\left(\mathrm{MFDFA}_{\mathrm{emd}}\right)$ detrending procedures (see technical details in Appendix 1). 


\section{Entropy analysis}

Entropy analysis (Chhabra \& Jensen, 1989) defines the scaledependent measure as a probability measure of the total magnitude of the response time fluctuation (i.e., sum of $x_{t}$ ) within the nonoverlapping time interval $[(v-1) s+1, v s]$ :

$\mu_{s, v}=\frac{\sum_{t=(v-1) s+1}^{v s} x_{t}}{\sum_{t=1}^{N} x_{t}}$

The definition of $\mu_{s, v}$ in entropy analysis contains no detrending procedure and, therefore, assumes that the response time series is a stationary process.

\section{Leader wavelet transformation}

Leader wavelet transformation (LWT; Wendt, 2008) defines the scale-dependent measure $\mu_{s, v}$ by a recursive high-pass and low-pass filtering procedure of the response time series $x_{t}$ illustrated in Fig. 2a. The scale-dependent measure $\mu_{s=2, v}$ on the smallest scale $s=2$ is the convolution product $\otimes$ of response time series $x_{t}$ and the high-pass filter $\psi_{t}^{h i}$ subsampled by 2 . The subsampled convolution product $\otimes$ of the response time series $x_{t}$ and low-pass filter $\psi_{t}^{l o}$ are used in another convolution and subsample procedure to define the statistical measure $\mu_{s, v}$ on the coarser scales $s=4$. The detrending of the response time series in LWT is dependent on the choice of $\psi_{t}^{h i}$ and $\psi_{t}^{l o}$ where the present study uses a third-order Daubechies filter (Daubechies, 1992). The subsampling by 2 leads to dyadic intervals $\lambda_{s, v}$ that double the sample sizes $s=2^{j}$ for the nonoverlapping intervals (width of the boxes in Fig. 2b) for each new level $j$, but halves the frequency band $f=1 / 2^{j}$ (height of the boxes in Fig. 2b). The local maximum of $\mu_{s, v}$ (i.e., wavelet leader illustrated in Fig. 2b) is used as a scale-dependent measure to prevent a degeneration of the multifractal spectrum by $\mu_{s, v} \rightarrow 0$.

\section{Gradient modulus wavelet projection}

Gradient modulus wavelet projection (GMWP; Turiel, PerezVicente, \& Grazzini, 2006) defines a scale-dependent measure by a continuous wavelet transformation:

$\mu_{s, t_{0}}=\sum_{t=t_{0}-s / 2}^{t_{0}+s / 2}\left|x_{t}\right| \psi_{s, t_{0}-t}$

The continuous wavelet transformation is the convolution of the response time series and a waveform $\psi_{s, t_{0}-t}$ scaled to the floating time interval $\left[t_{0}-s / 2, t_{0}+s / 2\right]$ (Mallat, 1999). The detrending of the response series is dependent on the shape of the waveform. The present study used a Laplacian wavelet $\psi_{s, t_{0}-t}=\left(1+\left(t^{2} / s\right)\right)^{-2}$ that performs well on the smallest scales (Turiel \& Perez-Vicente, 2003).

\section{Local detrended fluctuation analyses}

Local detrended fluctuation analyses $\left(\mathrm{DFA}_{\text {loc }}\right.$; Ihlen, 2012) defines the scale-dependent measure $\mu_{s, t_{0}}$ as a
Fig. 2 Schematic

representation of the leader wavelet transformation. a The discrete wavelet transformation computes the statistical measure $\mu_{s, v}$ by recursive high-pass and low-pass filtering procedure of the response time series $x_{t}$. $\mathbf{b}$ Twenty-eight samples of a time series (upper panel) and the dyadic intervals $\mu_{s, v}$ of the statistical measure $\mu_{s, v}$ (lower panel) defined by the recursive filtering procedure. The wavelet leaders is defined as the local maxima of three subsequent intervals $\lambda_{s^{\prime}, v}$ on scale $s^{\prime}$ and for all $s<s^{\prime}$ (gray colored boxes)

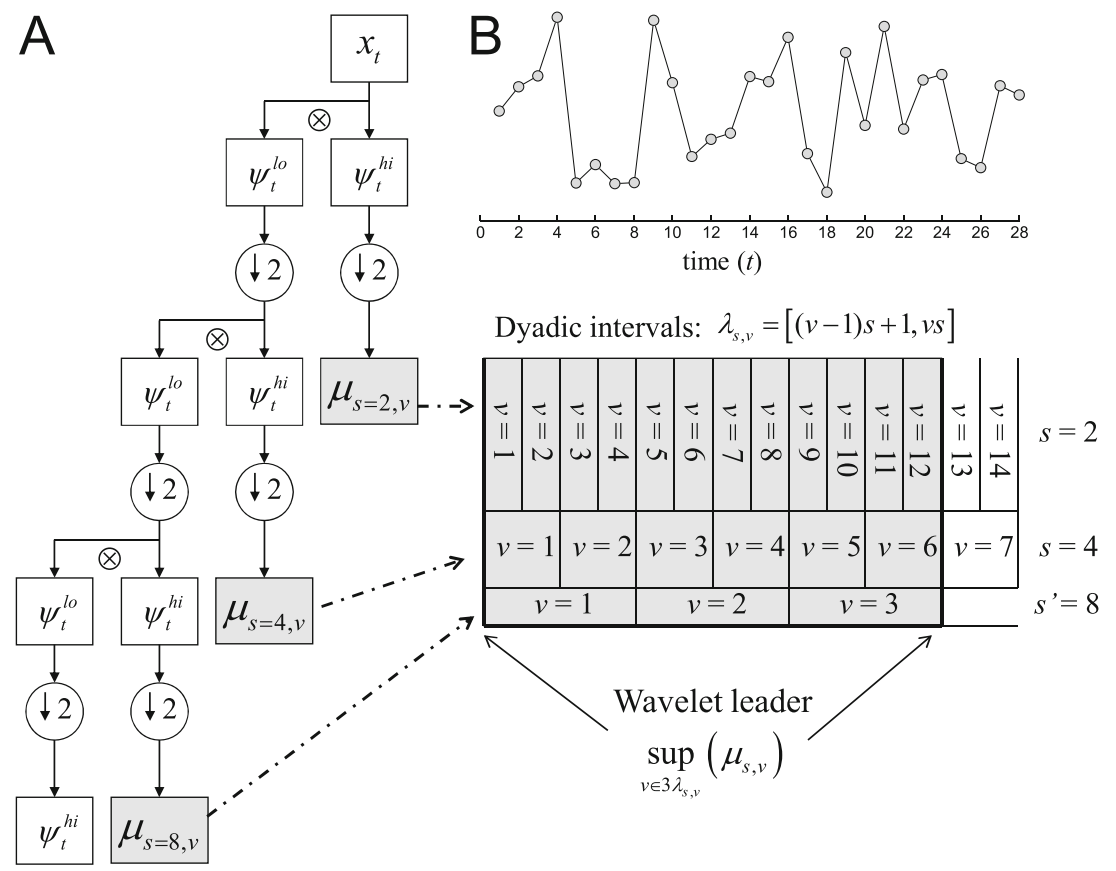


root-mean square fluctuation of the integrated response time series $y_{t}=\sum_{i=1}^{t}\left(x_{i}-\bar{x}\right)$ around a polynomial trend $\widehat{y}_{t, m}$ of order $m$ within a floating trial interval $\left[t_{0}-s / 2, t_{0}+s / 2\right]$ :

$\mu_{s, t_{0}}=\sqrt{\frac{1}{s} \sum_{t=t_{0}-s / 2}^{t_{0}+s / 2}\left[y_{t}-\hat{y}_{t, m}\right]^{2}}$

The present study used a second-order polynomial detrending (i.e., $m=2$ ) of the response time series.

Indirect estimation from $q$-order statistics of $\mu_{s, v}$

The $q$-order statistical moments $E\left(\mu_{s, v}^{q}\right)$ are defined for the scale-dependent measure of MFDFA and LWT by the following equation (Parisi \& Frisch, 1985):

$E\left(\mu_{s, v}^{q}\right)=\frac{1}{N_{s}} \sum_{v=1}^{N_{s}} \mu_{s, v}^{q} \propto s^{\zeta_{q}}$

where $N_{s}$ is the number of nonoverlapping intervals at each scale $s . E\left(\mu_{s, v}^{q}\right)$ satisfies the power law on the right-hand side when the response time series has a multifractal structure. The $q$-order scaling exponent $\zeta_{q}$ is estimated as the linear regression slope of $E\left(\mu_{s, v}^{q}\right)$ versus $s$ in log-log coordinates (see middle left panel in Fig. 1). Next, the $q$-order singularity exponent $h_{q}$ and singularity dimension $D_{q}$ are defined by the following Legendre transformation of $\zeta_{q}$ (Parisi \& Frisch, 1985):

$$
\begin{aligned}
h_{q} & =\frac{d \zeta_{q}}{d q} \\
D_{q} & =1+q h_{q}-\zeta_{q}
\end{aligned}
$$

Finally, the multifractal spectrum $D_{h}$ is defined by plotting $h_{q}$ versus $D_{q}$ (see lower left panel in Fig. 1).

In entropy analysis, the scale-dependent measure of Eq. 2 is used to estimate $h_{q}$ and $D_{q}$ directly from the following $q$-order entropies $F_{h}\left(\mu_{s, v}^{q}\right)$ and $F_{D}\left(\mu_{s, v}^{q}\right)$, respectively, without the Legendre transformation of Eq. 6 (Chhabra \& Jensen, 1989):

$$
\begin{gathered}
F_{h}\left(\mu_{s, v}^{q}\right)=\sum_{v=1}^{N_{s}} P_{s, v}^{q} \log \left(\mu_{s, v}\right) \propto s^{h_{q}} \\
F_{D}\left(\mu_{s, v}^{q}\right)=\sum_{v=1}^{N_{s}} P_{s, v}^{q} \log \left(P_{s, v}^{q}\right) \propto s^{D_{q}}
\end{gathered}
$$

where

$$
P_{s, v}^{q}=\frac{\mu_{s, v}^{q}}{\sum_{v=1}^{N_{s}} \mu_{s, v}^{q}}
$$

Similar to the estimation of $\zeta_{q}$ in MFDFA and LWT, $h_{q}$ and $D_{q}$ are estimated as the linear regression slopes of the $q$ - order entropies $F_{h}\left(\mu_{s, v}^{q}\right)$ and $F_{D}\left(\mu_{s, v}^{q}\right)$, respectively, and scale $s$ in log-log coordinates.

Direct estimation from $\mu_{s, t_{0}}$

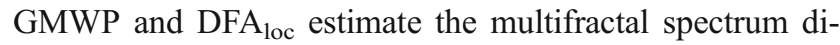
rectly from the scale-dependent measure of Eqs. 3 and 4, respectively. The temporal variation in the trial dependency of a response time series can be defined by the local singularity exponents $h_{t_{0}}$ when scale-dependent measure $\mu_{s, t_{0}}$ satisfies the following power law (Canus, Lévy Véhel, \& Tricot, 1998, Turiel, Yahia, \& Pérez-Vicente, 2008):

$\mu_{s, t_{0}} \propto \lim _{s \rightarrow 0} s^{h_{t_{0}}}$

The local scaling exponent $h_{t}$ is estimated as the linear regression slope of log-log plot of the scale-dependent measure $\mu_{s, t_{0}}$ versus scale $s$ (see the middle right panel in Fig. 1), but the present study uses a method suggested by Struzik (2000) that provides more numerically stable results (see technical details in Appendix 2). The multifractal spectrum $D_{h}$ is defined by the normalized distribution $P_{h}$ of $h_{t_{0}}$ in $\log$ coordinates by the following equation (Turiel et al., 2008):

$D_{h}=\lim _{\varepsilon \rightarrow 0}\left(1-\frac{\log \left(P_{h} / P_{h}^{\max }\right)}{\log (\varepsilon)}\right)$

where $\varepsilon$ is the bin size of the histogram used to define $P_{h}$ and $P_{h}^{\max }$ is the maximum probability at the mode $\bar{h}$ of $h_{t_{0}}$ (see the lower right panel in Fig. 1). The most common trial dependency in the response series is the mode $\bar{h}$ and is assigned $D_{h}=1$ by Eq. 9 .

In summary, the present article compares the performance of the seven multifractal analyses represented in Table 1 and defined by the choice of scale-dependent measure and $q$-order statistics. Table 1 also summarizes the parameter settings used to compare the performance of the multifractal analyses. All multifractal analyses in Table 1 were conducted in MATLAB and are available at www.ntnu.edu/inm/geri/software.

\section{Multiplicative cascading noise}

The seven multifractal analyses represented in Table 1 were tested on the stationary increments $\Delta B_{H}(A(t))=B_{H}(A(t+1))-$ $B_{H}(A(t))$ of a multifractal random walk $B_{H}(A(t))$ (Bacry, Muzy, $\&$ Delour, 2001; Ihlen \& Vereijken, 2010). The main feature of $\Delta B_{H}(A(t))$ is intermittent periods with variation of large and small magnitudes (see red and blue intervals, respectively, in Fig. 3). The increasing width of the multifractal spectrum $D_{h}$ of $\Delta B_{H}(A(t))$ reflects increasing differences in the magnitudes of intermittent small and large variations (compare the variations within the red and blue intervals for light to dark blue traces in Fig. 3). The central tendency $\bar{h}$ reflects the long-range 
Table 1 Summary of definitions and parameter settings for the seven multifractal analyses compared in the present study

\begin{tabular}{|c|c|c|c|c|}
\hline Analysis (abbrivation) & Formalism & Measure $\mu_{s, t}$ & Scaling range & $q$-range \\
\hline MFDFA & Eqs. 5 and 6 & Eq. 1 & $16 \leq s \leq N / 4$ & $-3 \leq q \leq 3$ \\
\hline MFDFA $_{\text {ma }}$ & Eqs. 5 and 6 & Eqs. 14 and 15 & $16 \leq s \leq N / 4$ & $-3 \leq q \leq 3$ \\
\hline MFDFA $_{\text {emd }}$ & Eqs. 5 and 6 & Eqs. 14 and 16 & $16 \leq s \leq N / 4$ & $-3 \leq q \leq 3$ \\
\hline Entropy & Eq. $7 \mathrm{a}$ & Eq. 2 & $16 \leq s \leq N / 4$ & $-3 \leq q \leq 3$ \\
\hline LWT & Eqs. 5 and 6 & Fig. 2 & $16 \leq s \leq N / 4$ & $-3 \leq q \leq 3$ \\
\hline GMWP & Eqs. 8 and 9 & Eq. 3 & $7 \leq s \leq 17$ & - \\
\hline $\mathrm{DFA}_{\mathrm{loc}}$ & Eqs. 8 and 9 & Eq. 4 & $7 \leq s \leq 17$ & - \\
\hline
\end{tabular}

dependency $H$ of the fractional Brownian motion $B_{H}(t)$. The present performance comparison utilized an algorithm developed by Chainais, Riedi, and Abry (2005) to generate 100 realizations of $\Delta B_{H}(A(t))$ of 1,024 and 4,096 samples with $H=$ $0.5,0.6,0.7,0.8$, and 0.9 and with a multifractal spectrum width $h_{\max }-h_{\min }=0,0.1,0.2,0.3,0.4,0.5$, and 0.6. A symmetric shape of the multifractal spectrum was provided by a lognormal cascade $A(t)$ constructed by random multipliers with a lognormal distribution.

Response time series are influenced by noise and nonstationary trends caused by differences in task difficulty, external perturbations, or internal mental strategies. Thus, Gaussiandistributed random noise with a signal-to-noise ratio of either 0.33 or 1 was added to $\Delta B_{H}(A(t))$ to test for the robustness of the seven multifractal analyses shown in Table 1 to additive noise (Delignières et al., 2006). Furthermore, a complex multiharmonic trend $T(t)$ given by the following equation was added to $\Delta B_{H}(A(t))$ to test the robustness of the multifractal analyses to complex additive trends:

$$
\begin{aligned}
T(t)= & C \sin (0.1250 \pi t)+C \sin (0.0313 \pi t) \\
& +C \sin (0.0078 \pi t)+C \sin (0.0020 \pi t)
\end{aligned}
$$

where $t$ is the sample number of $\Delta B_{H}(A(t))$. The amplitudes $C$ of oscillations were 0.33 and 1 times the root-mean square variation of $\Delta B_{H}(A(t))$ (i.e., signal-to-trend ratios of 0.33 and 1). Figure 4 illustrates $\Delta B_{H}(A(t))$ with $H=0.9$ (see top panel) with additive white noise (see red trace in left panels) and multiharmonic trend

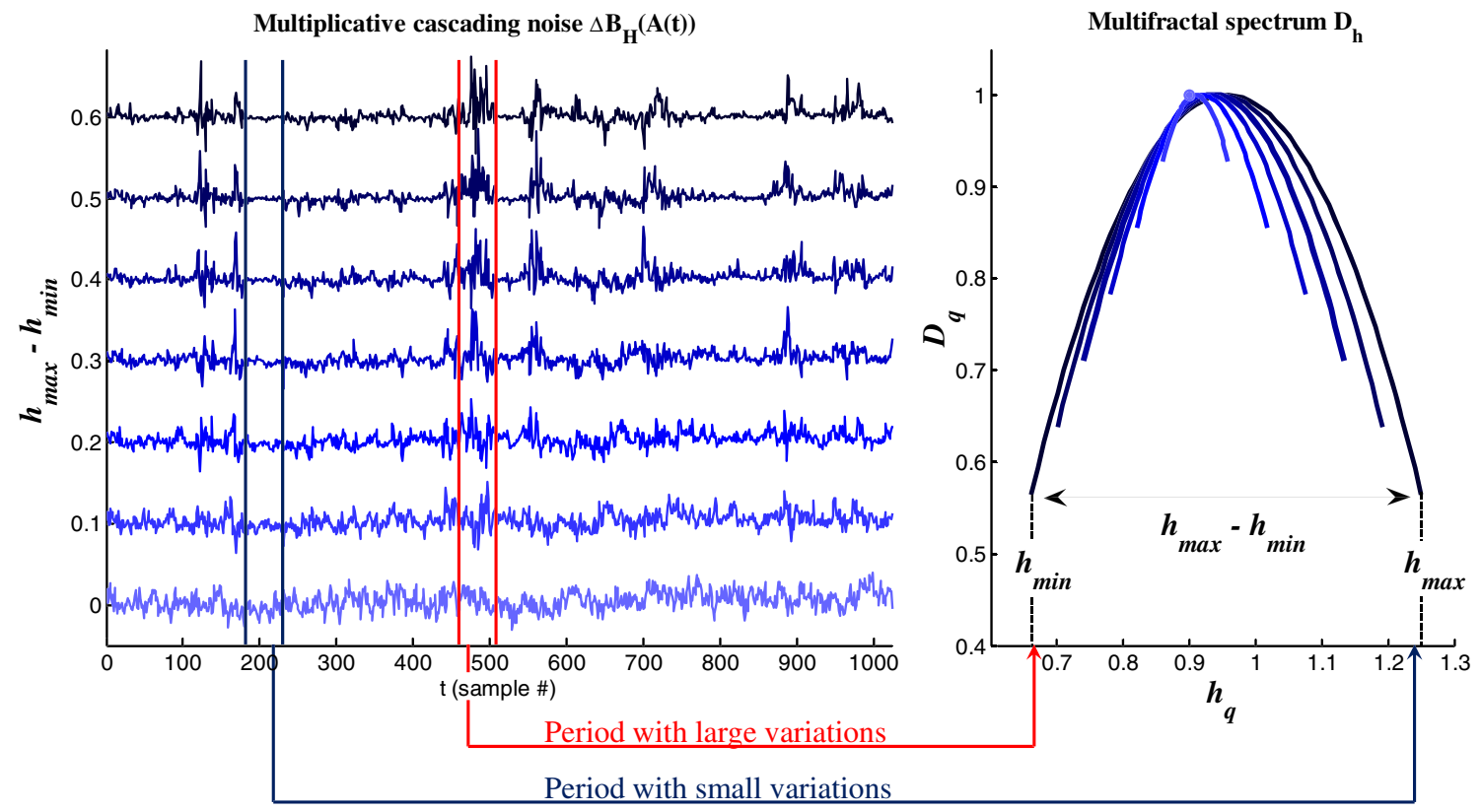

Fig. 3 Intermittent variations in the multifractal process $\Delta B_{H}(A(t))$ with $H=0.9$ for the seven multifractal spectrum widths $h_{\max }-h_{\min }$ used in the present comparative study of multifractal analyses (traces from light to dark blue in the left panel with corresponding colored spectrum in the right panel). $h_{\max }-h_{\min }$ are directly related to the width of the distribution of interaction multipliers used to generate the cascade $A(t)$ within $\Delta B_{H}(A(t))$. The blue interval and arrow indicate the intermittent period of small variation with local scaling exponents in the right tail of $D_{h}$ close to $h_{\max }$. The red interval and arrow indicate intermittent period of large variation with local exponent in the left tail of $D_{h}$ close to $h_{\max }$. The difference between the magnitude of small and large variations within $\Delta B_{H}(A(t))$ increases with its multifractal spectrum width $h_{\max }-h_{\min }$ indicated by an increase in the large variation within the red intervals and a decrease in variation within the blue intervals 


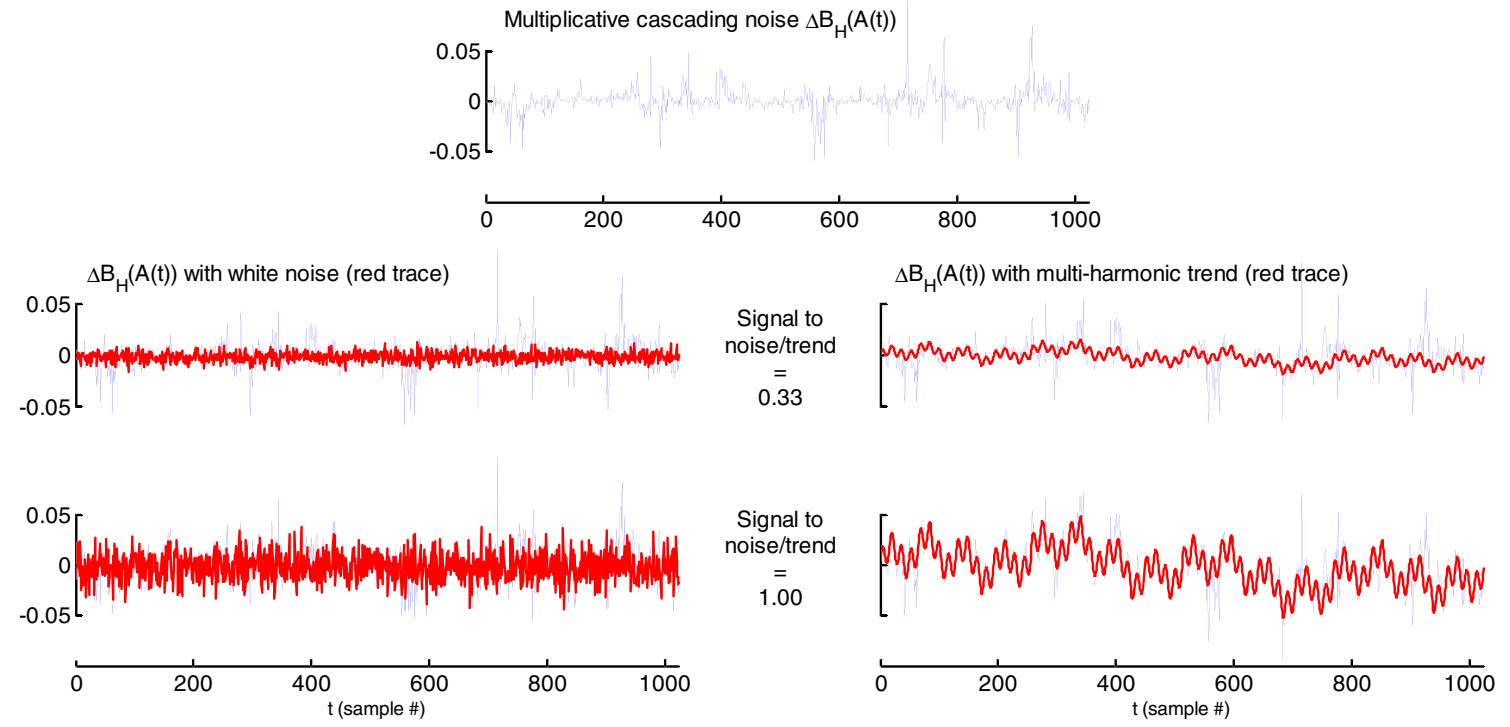

Fig. 4 Multiplicative cascading noise $\Delta B_{H}(A(t))$ with $H=0.9$ (top panel) with additive white noise and multiharmonic trend (red traces). Right panels show $\Delta B_{H}(A(t))$ with white noise with a moderate signalto-noise ratio of 0.33 (middle panel) and a high signal-to-noise ratio of

1 (bottom panel). Left panels show $\Delta B_{H}(A(t))$ with multiharmonic trends with a moderate signal-to-trend ratio of 0.33 (middle panel) and a high signal-to-trend ratio of 1 (bottom panel)

(see red trace in right panels). The white noise and multiharmonic trend generate minor distortions of the intermittent variation in $\Delta B_{H}(A(t))$ for the signal-to-noise/trend ratio of 0.33 (see middle panels in Fig. 4), whereas major distortion of the intermittent variation in $\Delta B_{H}(A(t))$ is generated for the signal-to-noise/trend ratio of 1 (see bottom panels in Fig. 4).

Performance comparison of the multifractal analyses

The performance of the multifractal analyses in Table 1 was evaluated by two error measures. The following error measure defines the deviations in the width and shape of the estimated multifractal spectrum $D_{h}^{e}$ and the predefined $D_{h}$ of $\Delta B_{H}(A(t))$ (Turiel et al., 2006):

$E_{D}=\frac{\left\langle\left|D_{h}-D_{h}^{e}\right|\right\rangle_{h}}{h_{\max }-h_{\min }}$

where $\langle|\ldots|\rangle_{h}$ defines the mean absolute difference between $D_{h}$ and $D_{h}^{e}$ and is taken over all values $h$ of $D_{h}$ and

normalized to the width $h_{\max }-h_{\min }$ of $D_{h}$. $D_{h}^{e}$ was set to zero for the $h$ of the model where the estimated $D_{h}^{e}$ has no defined value (Turiel et al., 2006). Furthermore, the estimated central tendency $\bar{h}^{e}$ and the central tendency of the model $\bar{h}$ were aligned (i.e., $\bar{h}^{e}=\bar{h}$ ) to exclude the influence of errors in estimated $\bar{h}^{e}$ on the error measure in Eq. 11. The root-mean square error of the estimated central tendency $\bar{h}^{e}$ was defined by the following equation:

$E_{\bar{h}}=\sqrt{\left\langle\left(\bar{h}-\bar{h}^{e}\right)^{2}\right\rangle}$

where $\bar{h}$ is the central tendency of model $D_{h}$ and $\bar{h}^{e}$ is the estimated central tendency by multifractal analyses in Table 1. The mean $\langle\ldots\rangle$ was computed for the entire ensemble of 100 model series of $\Delta B_{H}(A(t))$ for each parameter setting. The following color convention was used to improve the readability of the tabulated results in the Results section below (Turiel et al., 2006):
$E \leq 0.10$
$0.10<E \leq 0.20$
$0.20<E \leq 0.30$
$E>0.30$

White box

Yellow box

Orange box

A paired sample $t$-test was employed to identify a significantly different $E_{D}$. If one or two analyses had a significantly smaller or larger $E_{D}(p<.05)$, as compared with the rest of the analyses, then an asterisk $*$ and a crucifix $\dagger$, respectively, was 
marked behind value. Significant differences for two analyses were also considered because the performance of MFDFA $_{\text {lin }}$ and MFDFA $A_{\text {ma }}$ together with DFA $_{\text {loc }}$ and GMWP were expected to be more related than the other analyses. The error measure $E_{D}$ was also visually evaluated with the mean and standard deviation of $D_{h}$ from the ensemble of 100 series of $\Delta B_{H}(A(t))$ where $D_{h}$ was aligned at their central tendency.

\section{Results}

Comparison of the estimated $D_{h}$

Figure 5 shows the mean $D_{h}$ and \pm 1 standard deviation (see bars) estimated by the seven multifractal analyses in Table 1 for the ensemble of $\Delta B_{H}(A(t))$ with $H=0.5$ and $h_{\max }-h_{\min }=0$ (see red bars) and $h_{\max }-h_{\min }=0.6$ (see blue bars). The best performance of analyses based on $q$-order statistics (i.e., MFDFA, LWT, and entropy analysis) was provided by MFDFA $_{\text {lin }}$ and MFDFA $A_{\text {ma }}$ when the multifractal analyses were employed to $\Delta B_{H}(A(t))$ with $H=0.5$ (see Table 2). However, the LWT and entropy analyses performed more consistently across $H$ and were superior to the MFDFAs for $H=0.9$, as indicated by error measures in Table 3. The LWT and entropy analyses were also superior to the GMWP and $\mathrm{DFA}_{\text {loc }}$ that showed similar inconsistency across $H$ as the MFDFAs. Furthermore, LWT had more consistent errors (i.e., systematic underestimation of $D_{h}$ ), as compared with the other analyses, based on the $q$-order statistics indicated in Fig. 5 by the smaller standard deviation for the left tail of the multifractal spectrum $D_{h}$.

Comparison of the estimated central tendency $\bar{h}$ of $D_{h}$

All multifractal analyses estimated the central tendency $\bar{h}$ of the multifractal spectrum with high precision. LWT had the largest estimation error $E_{\bar{h}}$, whereas the GMWP had the smallest $E_{\bar{h}}$ for both sample sizes, as indicated in Table 4. However, GMWP had larger variation in $\bar{h}$, as compared with $\mathrm{DFA}_{\text {loc }}$. The estimation of central tendency was consistent across $H$ of $\Delta B_{H}(A(t))$ but was influenced by the presence of white noise and multiharmonic trends.

\section{Influence of sample size}

The error measure $E_{D}$ decreased in magnitude and was more consistent with an increased sample size (i.e., $N=4,096$ ) for all multifractal analyses. In addition, all analyses performed more consistently across $H$ of $\Delta B_{H}(A(t))$, and the differences between the performance of multifractal analyses decreased. The largest decrease in magnitude of $E_{D}$ was observed for $\mathrm{MFDFA}_{\text {emd }}$, where the performance became comparable to those for MFDFA lin and MFDFA ma $_{\text {(compare upper and }}$ lower parts of Table 2). Nevertheless, MFDFA ${ }_{\text {emd }}$ had less
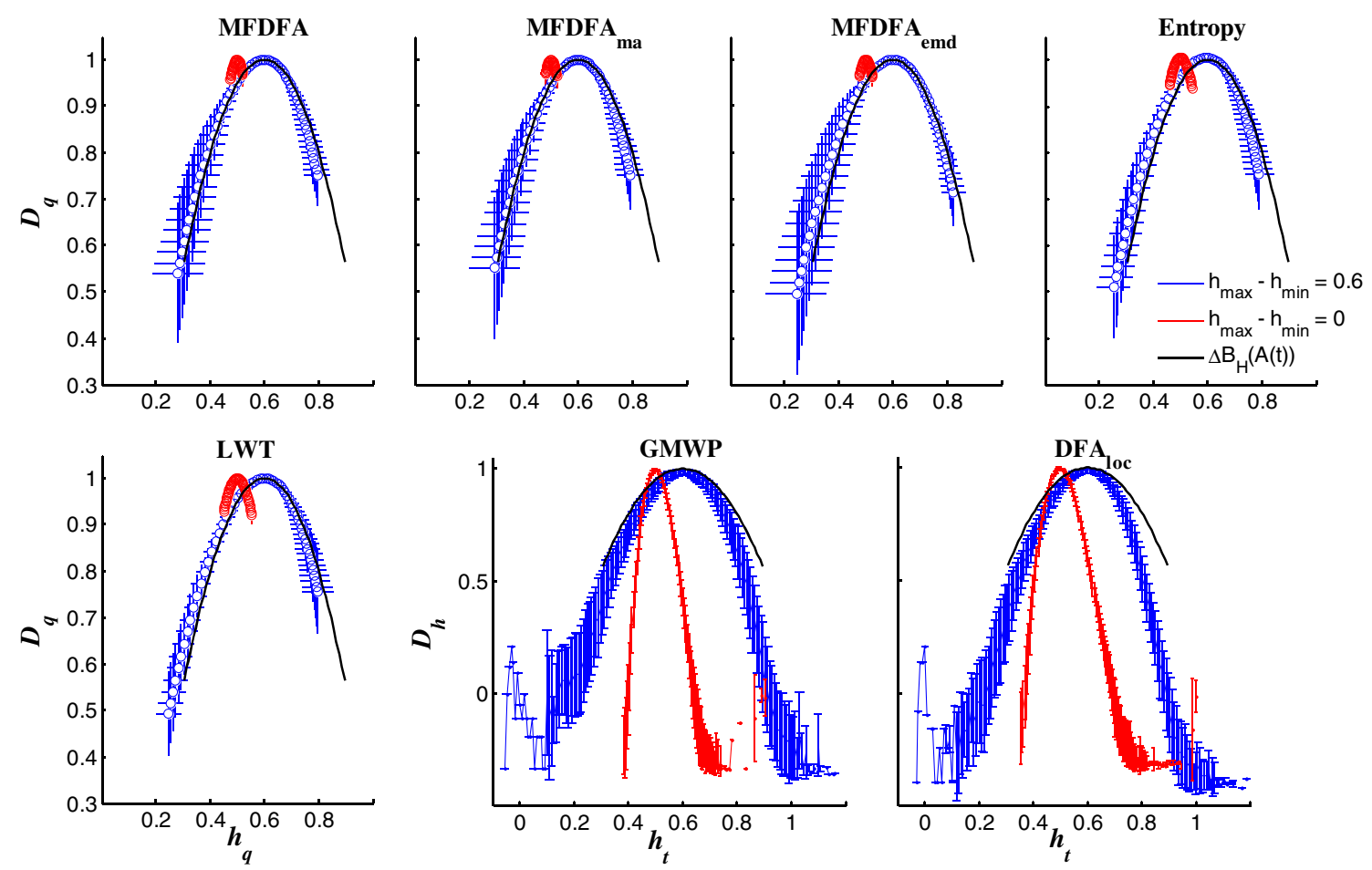

Fig. 5 Mean $D_{h}$ and $\pm 1 S D$ (bars) estimated by the seven multifractal analyses in Table 1 for the ensemble of $\Delta B_{H}(A(t))$ with 4,096 samples and $h_{\max }-h_{\min }=0$ (red bars) and $h_{\max }-h_{\min }=0.6$ (blue bars) 
Table 2 Mean error $E_{D}$ for six different multifractal spectrum widths $h_{\max }-h_{\min }$ for $\Delta B_{H}(A(t))$ with $H=0.5$ and with no noise or trends present

\begin{tabular}{|c|c|c|c|c|c|c|c|}
\hline & \multicolumn{7}{|c|}{$h_{\max }-h_{\min }$} \\
\hline & 0 & 0.1 & 0.2 & 0.3 & 0.4 & 0.5 & 0.6 \\
\hline & \multicolumn{7}{|c|}{$N=1024$} \\
\hline MFDFA & $0.08^{*}$ & $0.10^{*}$ & $0.14 *$ & $0.14^{*}$ & 0.14 & 0.10 & 0.09 \\
\hline MFDFA $_{\mathrm{ma}}$ & $0.07 *$ & $0.09 *$ & $0.14 *$ & $0.14 *$ & 0.14 & 0.10 & 0.09 \\
\hline MFDFA $_{\text {emd }}$ & 0.10 & 0.12 & 0.17 & 0.16 & 0.14 & $0.12 \dagger$ & $0.13 \dagger$ \\
\hline Entropy & 0.11 & 0.13 & 0.17 & 0.15 & 0.12 & 0.09 & 0.07 \\
\hline LWT & 0.12 & 0.14 & 0.17 & 0.16 & 0.12 & 0.09 & 0.08 \\
\hline GMWP & $0.20 \dagger$ & $0.21 \dagger$ & 0.20 & 0.17 & 0.13 & 0.09 & $0.06^{*}$ \\
\hline \multirow{2}{*}{$\mathrm{DFA}_{\mathrm{loc}}$} & $0.20 \dagger$ & $0.21 \dagger$ & 0.20 & 0.17 & 0.13 & 0.09 & $0.06^{*}$ \\
\hline & \multicolumn{7}{|c|}{$N=4096$} \\
\hline MFDFA & 0.05 & 0.06 & 0.07 & 0.06 & 0.05 & 0.06 & $0.08 \dagger$ \\
\hline MFDFA $_{\mathrm{ma}}$ & 0.05 & 0.05 & 0.06 & 0.05 & 0.05 & 0.07 & $0.09 \dagger$ \\
\hline MFDFA $_{\text {emd }}$ & 0.06 & 0.06 & 0.06 & 0.07 & 0.07 & 0.06 & 0.05 \\
\hline Entropy & 0.08 & 0.09 & 0.09 & 0.08 & 0.05 & 0.03 & 0.05 \\
\hline LWT & 0.10 & 0.11 & 0.12 & 0.11 & 0.07 & 0.05 & 0.05 \\
\hline GMWP & $0.20 \dagger$ & $0.17 \dagger$ & $0.14 \dagger$ & 0.11 & 0.06 & 0.03 & 0.05 \\
\hline $\mathrm{DFA}_{\mathrm{loc}}$ & $0.20 \dagger$ & $0.17 \dagger$ & $0.14 \dagger$ & 0.11 & 0.06 & 0.03 & 0.05 \\
\hline
\end{tabular}

$\dagger, *$ represents a significantly larger and smaller mean error $E_{D}$ (paired $t$-test; $p<0.05$ )

consistent error $E_{D}$, as compared with the other analyses, indicated by large standard deviations in the tails of $D_{h}$ in Figs. 5 and 6. The largest increase in consistency of $E_{D}$ with sample size was observed in $\mathrm{DFA}_{\mathrm{loc}}$ that had a twofold lower standard deviation of $E_{D}$, as compared with the analyses based on the $q$ order statistics (i.e., MFDFA, LWT, and entropy analysis).

\section{Influence of additive noise}

Figure 6 shows the mean $D_{h}$ and $\pm 1 S D$ (see bars) estimated by the seven multifractal analyses in Table 1 for the ensemble of $\Delta B_{H}(A(t))$ with additive white noise. The blue, green, and red markers in Fig. 6 are the estimated $D_{h}$ for $\Delta B_{H}(A(t))$ with signal-to-noise ratios of $0,0.33$, and 1 , respectively. Comparison of blue, green, and red markers in Fig. 6 shows that the increasing magnitude of additive white noise truncates the estimated multifractal spectrum $D_{h}$ and translates its central tendency toward $\bar{h}=0.5$. This result was especially apparent for the MFDFA, LWT, and entropy analyses.

All multifractal analyses were quite robust to additive white noise in the case of the moderate signal-to-noise ratio of 0.33 , as is shown in Table 5. The robustness to noise appeared visually as the preservation of the left side (i.e., positive $q s$ ) of the multifractal spectra for the MFDFA, LWT, and entropy analyses (compare blue and green $D_{h}$ in Fig. 6). However, the same analyses yield the abovementioned changes for both the left and right sides of $D_{h}$ for large noise levels (i.e., signal-to-noise ratio of 1), as is shown by the increased error measure $E_{D}$ in the upper part of Table 6 and the truncated red multifractal spectra in Fig. 6. GMWP and DFA ${ }_{\text {loc }}$ had a more noise-resistant performance, as compared with the analyses based on the $q$ -

Table 3 Mean error $E_{D}$ for five different $H$ of $\Delta B_{H}(A(t))$ with multifractal spectrum width $h_{\max }-h_{\min }=0.6$ and simple sizes 1,024 (upper panel) and 4,096 (lower panel)

\begin{tabular}{|c|c|c|c|c|c|}
\hline & $H=0.5$ & $H=0.6$ & $H=0.7$ & $H=0.8$ & $H=0.9$ \\
\hline & \multicolumn{5}{|c|}{$N=1024, h_{\max }-h_{\min }=0.6$} \\
\hline MFDFA & 0.09 & 0.10 & 0.12 & 0.13 & 0.17 \\
\hline MFDFA $_{\text {ma }}$ & 0.09 & 0.11 & 0.11 & 0.13 & 0.13 \\
\hline MFDFA $_{\mathrm{emd}}$ & 0.07 & 0.09 & 0.10 & 0.12 & 0.14 \\
\hline Entropy & 0.07 & 0.09 & 0.11 & 0.11 & 0.12 \\
\hline LWT & 0.07 & 0.10 & 0.11 & 0.11 & 0.11 \\
\hline GMWP & 0.06 & 0.11 & 0.11 & 0.13 & 0.16 \\
\hline \multirow[t]{2}{*}{$\mathrm{DFA}_{\mathrm{loc}}$} & 0.06 & 0.11 & 0.11 & 0.13 & 0.16 \\
\hline & \multicolumn{5}{|c|}{$N=4096, h_{\max }-h_{\min }=0.6$} \\
\hline MFDFA & 0.08 & 0.05 & 0.06 & 0.08 & 0.09 \\
\hline MFDFA $_{\mathrm{ma}}$ & 0.09 & 0.06 & 0.06 & 0.07 & 0.07 \\
\hline MFDFA $_{\text {emd }}$ & 0.05 & 0.04 & 0.06 & 0.07 & 0.08 \\
\hline Entropy & 0.05 & 0.04 & 0.05 & 0.06 & 0.06 \\
\hline LWT & 0.04 & 0.04 & 0.06 & 0.07 & 0.06 \\
\hline GMWP & 0.02 & 0.05 & 0.06 & 0.08 & 0.09 \\
\hline $\mathrm{DFA}_{\text {loc }}$ & 0.02 & 0.05 & 0.06 & 0.08 & 0.09 \\
\hline
\end{tabular}


Table 4 Error $E_{\bar{h}}$ of the position of the central tendency for $\Delta B_{H}(A(t))$ with five different $H$ and multifractal spectrum width $h_{\max }-h_{\min }=0.6$ and sample sizes 1,024 (upper part) and 4,096 (lower part)

\begin{tabular}{|c|c|c|c|c|c|}
\hline & $H=0.5$ & $H=0.6$ & $H=0.7$ & $H=0.8$ & $H=0.9$ \\
\hline & \multicolumn{5}{|c|}{$N=1024, h_{\max }-h_{\min }=0.6$} \\
\hline MFDFA & 0.08 & 0.09 & 0.08 & 0.08 & 0.10 \\
\hline MFDFA $_{\text {ma }}$ & 0.09 & 0.09 & 0.09 & 0.09 & 0.09 \\
\hline MFDFA $_{\text {emd }}$ & 0.08 & 0.08 & 0.08 & 0.08 & 0.10 \\
\hline Entropy & 0.09 & 0.09 & 0.05 & 0.01 & 0.06 \\
\hline LWT & 0.09 & 0.11 & 0.12 & 0.14 & 0.15 \\
\hline GMWP & 0.01 & 0.03 & 0.01 & 0.01 & 0.01 \\
\hline \multirow[t]{2}{*}{$\mathrm{DFA}_{\mathrm{loc}}$} & 0.08 & 0.08 & 0.06 & 0.07 & 0.08 \\
\hline & \multicolumn{5}{|c|}{$N=4096, h_{\max }-h_{\min }=0.6$} \\
\hline MFDFA & 0.04 & 0.04 & 0.04 & 0.04 & 0.04 \\
\hline MFDFA $_{\text {ma }}$ & 0.04 & 0.04 & 0.05 & 0.05 & 0.05 \\
\hline MFDFA $_{\text {emd }}$ & 0.04 & 0.04 & 0.04 & 0.04 & 0.04 \\
\hline Entropy & 0.05 & 0.04 & 0.02 & 0.01 & 0.07 \\
\hline LWT & 0.06 & 0.07 & 0.08 & 0.10 & 0.11 \\
\hline GMWP & 0.01 & 0.01 & 0.01 & 0.02 & 0.02 \\
\hline $\mathrm{DFA}_{\text {loc }}$ & 0.05 & 0.05 & 0.05 & 0.05 & 0.05 \\
\hline
\end{tabular}

order statistics, for both the small $N=1,024$ and large $N=$ 4,096 sample sizes, especially for large multifractal spectrum widths $h_{\max }-h_{\min }=0.5$ and 0.6. However, the red traces in Fig. 6 for GMWP and DFA $\mathrm{loc}_{\mathrm{loc}}$ are comparable to the red monofractal traces in Fig. 5 and indicate that the superior performance of GMWP and $\mathrm{DFA}_{\text {loc }}$ is related to their overestimation of the monofractal spectrum width.
The estimation of the central tendency $\bar{h}$ of $D_{h}$ was quite robust to the moderate signal-to-noise ratio of 0.33 , but the MFDFA, LWT, and entropy analyses showed less consistency across the different $H$ of $\Delta B_{H}(A(t))$, as compared with GMWP and $\mathrm{DFA}_{\mathrm{loc}}$, for the large signal-to-noise ratio of 1 (see red traces in Fig. 6 and comparison of MFDFA emd $_{\text {and }}$ and GMWP in the upper part of Table 7). GMWP was the most
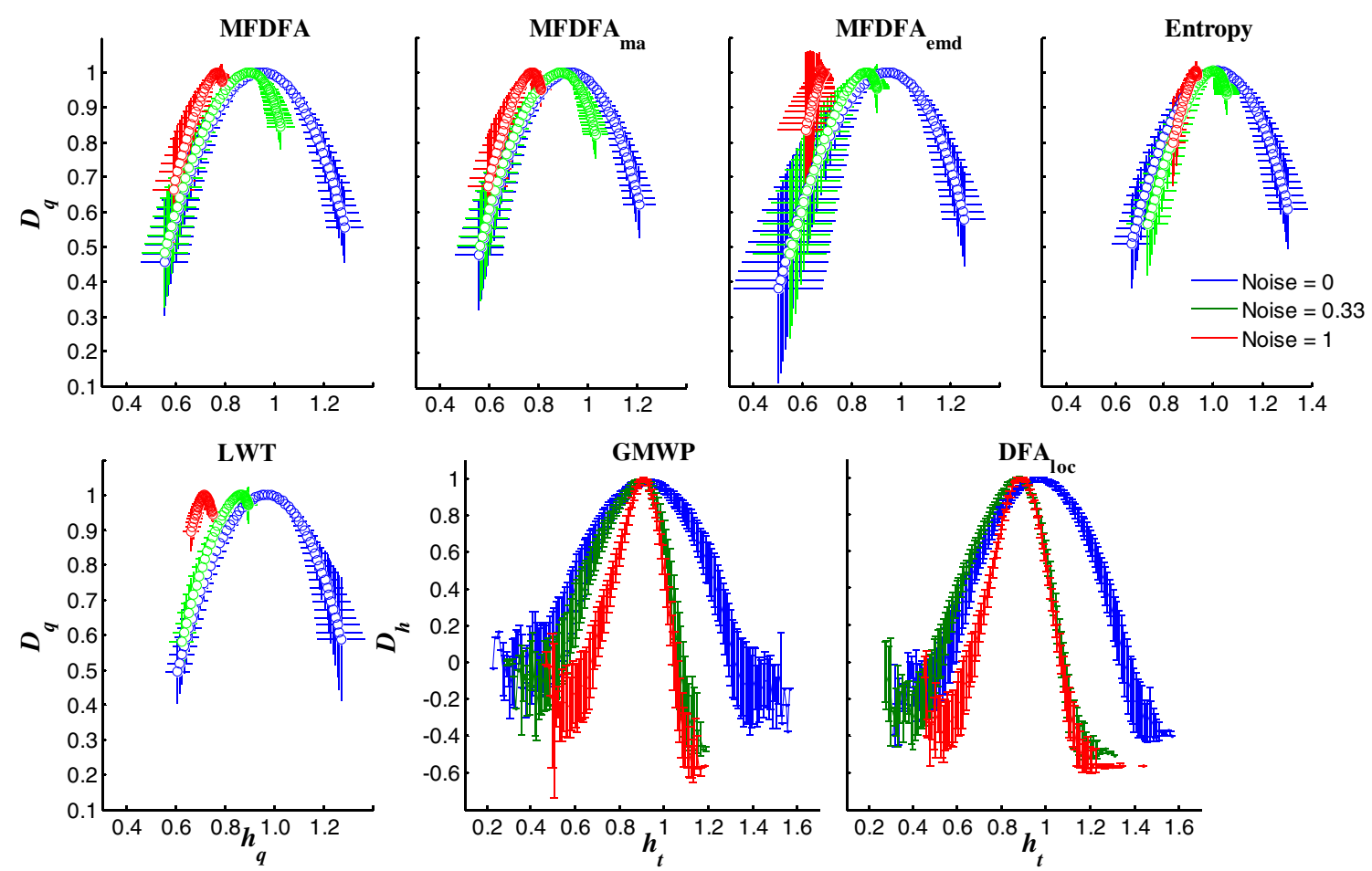

Fig. 6 Mean $D_{h}$ and $\pm 1 S D$ (bars) estimated by the seven multifractal analyses in Table 1 for the ensemble of $\Delta B_{H}(A(t))$ with white noise. The estimated $D_{h}$ represented for no additive noise (blue bars), a signal-to-

noise ratio of 0.33 (green bars), and a signal-to-noise ratio of 1 (red bars). The parameter settings for $\Delta B_{H}(A(t))$ are $h_{\max }-h_{\min }=0.6$ and $H=0.9$, and the sample size is 4,096 
Table 5 Mean error $E_{D}$ for six different multifractal spectrum widths $h_{\max }-h_{\min }$ for $\Delta B_{H}(A(t))$ with $H=0.5$ and with signal-to-noise ratio $=0.33$, but with no trends present

\begin{tabular}{|c|c|c|c|c|c|c|c|}
\hline & \multicolumn{7}{|c|}{$h_{\max }-h_{\min }$} \\
\hline & 0 & 0.1 & 0.2 & 0.3 & 0.4 & 0.5 & 0.6 \\
\hline & \multicolumn{7}{|c|}{$\mathrm{N}=1024$, Signal-to-noise $=0.33$} \\
\hline MFDFA & $0.09 *$ & 0.09 & 0.09 & 0.09 & 0.09 & 0.11 & 0.14 \\
\hline MFDFA $_{\mathrm{ma}}$ & $0.09 *$ & $0.08 *$ & 0.09 & 0.08 & 0.10 & 0.12 & 0.14 \\
\hline MFDFA $_{\text {emd }}$ & 0.11 & 0.11 & 0.12 & 0.11 & 0.10 & 0.12 & 0.13 \\
\hline Entropy & 0.11 & 0.11 & 0.10 & 0.07 & 0.06 & 0.07 & 0.11 \\
\hline LWT & 0.12 & 0.12 & 0.10 & 0.09 & 0.07 & 0.07 & 0.11 \\
\hline GMWP & $0.20 \dagger$ & $0.19 \dagger$ & $0.16 \dagger$ & 0.12 & 0.07 & $0.03 *$ & $0.05^{*}$ \\
\hline \multirow[t]{2}{*}{$\mathrm{DFA}_{\mathrm{loc}}$} & $0.20 \dagger$ & $0.19 \dagger$ & $0.16 \dagger$ & 0.12 & 0.07 & $0.03 *$ & $0.05 *$ \\
\hline & \multicolumn{7}{|c|}{$\mathrm{N}=4096$, Signal-to-noise $=0.33$} \\
\hline MFDFA & 0.05 & 0.04 & 0.04 & 0.05 & 0.07 & 0.12 & 0.15 \\
\hline MFDFA $_{\text {ma }}$ & 0.05 & $0.03 *$ & 0.04 & 0.06 & 0.08 & 0.12 & 0.14 \\
\hline MFDFA $_{\text {emd }}$ & 0.05 & 0.04 & 0.04 & 0.05 & 0.07 & 0.10 & 0.12 \\
\hline Entropy & 0.08 & 0.06 & 0.04 & 0.03 & 0.04 & 0.08 & 0.13 \\
\hline LWT & 0.10 & 0.08 & 0.06 & 0.04 & 0.05 & 0.09 & 0.14 \\
\hline GMWP & $0.20 \dagger$ & $0.17 \dagger$ & $0.14 \dagger$ & $0.11 \dagger$ & 0.06 & $0.03 *$ & $0.05 *$ \\
\hline $\mathrm{DFA}_{\text {loc }}$ & $0.20 \dagger$ & $0.17 \dagger$ & $0.14 \dagger$ & $0.11 \dagger$ & 0.06 & $0.03 *$ & $0.05^{*}$ \\
\hline
\end{tabular}

$\dagger, *$ represents a significantly larger and smaller mean error $E_{D}$ (paired $t$-test; $p<0.05$ )

robust analysis to the presence of additive white noise when estimating the central tendency $\bar{h}$ of $D_{h}$.

Influence of additive multiharmonic trends

Figure 7 shows the mean $D_{h}$ and $\pm 1 S D$ (see bars) estimated by the seven multifractal analyses in Table 1 for the ensemble of $\Delta B_{H}(A(t))$ with additive multiharmonic trends. The blue, green, and red markers in Fig. 6 are the estimated $D_{h}$ for $\Delta B_{H}(A(t))$ with signal-to-trend ratios of $0,0.33$, and 1 , respectively. Comparison of blue, green, and red markers in Fig. 7 indicates that the increasing magnitude of multiharmonic trends truncates the estimated multifractal spectrum $D_{h}$ and translates its central tendency towards $\bar{h}=1$.
Most analyses were quite robust to the moderate signalto-trend ratio of 0.33 , as is indicated by less truncation in the green spectra, as compared with the red spectra, in Fig. 7. $\mathrm{MFDFA}_{\text {lin }}$ and $\mathrm{MFDFA}_{\text {ma }}$ had the best performance when estimating the multifractal spectra with narrow spectrum widths (see stars in Table 8), but the performance declined for the large spectrum width by a truncation of the right side of $D_{h}$ (compare blue and green spectra in Fig. 7). GMWP and $\mathrm{DFA}_{\text {loc }}$ had the best performance when estimating multifractal spectra with wider spectrum widths (see stars in Table 8 and the lack of truncation in the green spectra in Fig. 7). However, entropy analysis had the most consistent performance across the range of multifractal spectrum widths, especially for the larger sample size of 4,096. The performance declined for the MFDFA, LWT, and entropy

Table 6 Mean error $E_{D}$ for seven different multifractal spectrum widths $h_{\max }-h_{\min }$ for $\Delta B_{H}(A(t))$ with $H=0.5$ and with signal-to-noise ratio $=1$ (upper part) and signal-to-trend ratio $=1$ (lower part)

\begin{tabular}{|c|c|c|c|c|c|c|c|}
\hline & \multicolumn{7}{|c|}{$h_{\max }-h_{\min }$} \\
\hline & 0 & 0.1 & 0.2 & 0.3 & 0.4 & 0.5 & 0.6 \\
\hline & \multicolumn{7}{|c|}{$N=1024$, Signal-to-Noise $=1$} \\
\hline MFDFA & 0.09 & 0.05 & 0.07 & 0.13 & 0.19 & 0.26 & 0.32 \\
\hline MFDFA $_{\text {ma }}$ & $0.08^{*}$ & 0.05 & 0.08 & 0.13 & 0.19 & 0.26 & 0.33 \\
\hline MFDFA $_{\mathrm{emd}}$ & 0.10 & 0.06 & 0.06 & 0.11 & 0.17 & 0.23 & 0.30 \\
\hline Entropy & 0.11 & 0.05 & 0.05 & 0.11 & 0.18 & 0.25 & 0.32 \\
\hline LWT & 0.12 & 0.05 & 0.04 & 0.10 & 0.18 & 0.24 & 0.29 \\
\hline GMWP & $0.20 \dagger$ & $0.13 \dagger$ & 0.07 & $0.03 *$ & $0.07 *$ & $0.14^{*}$ & $0.20^{*}$ \\
\hline \multirow[t]{2}{*}{$\mathrm{DFA}_{\mathrm{loc}}$} & $0.20 \dagger$ & $0.13 \dagger$ & 0.07 & $0.03 *$ & $0.07 *$ & $0.14^{*}$ & $0.20 *$ \\
\hline & \multicolumn{7}{|c|}{$N=1024$, Signal-to-Trend $=1$} \\
\hline MFDFA & 0.14 & 0.05 & 0.05 & 0.13 & 0.21 & 0.28 & 0.36 \\
\hline MFDFA $_{\mathrm{ma}}$ & 0.10 & $0.02 *$ & 0.09 & 0.17 & 0.25 & 0.32 & $0.40 \dagger$ \\
\hline MFDFA $_{\text {emd }}$ & 0.22 & 0.14 & $0.24 \dagger$ & $0.29 \dagger$ & $0.35 \dagger$ & 0.31 & 0.29 \\
\hline Entropy & 0.11 & 0.06 & 0.05 & $0.05^{*}$ & 0.09 & 0.15 & 0.21 \\
\hline LWT & $0.09 *$ & 0.07 & 0.05 & 0.07 & 0.12 & 0.16 & 0.21 \\
\hline GMWP & 0.23 & $0.19 \dagger$ & 0.15 & 0.09 & $0.04 *$ & $0.04 *$ & $0.09 *$ \\
\hline $\mathrm{DFA}_{\mathrm{loc}}$ & 0.23 & $0.19 \dagger$ & 0.15 & 0.09 & $0.04 *$ & $0.04 *$ & $0.09 *$ \\
\hline
\end{tabular}

$\dagger,{ }^{*}$ represents a significantly larger and smaller mean error $E_{D}$ (paired $t$-test; $p<0.05$ ) 
Table 7 Comparison of the error $E_{\bar{h}}$ of the position of the central tendency of MFDFA emd and GMWP for $\Delta B_{H}(A(t))$ with sample size 4,096 for five different $H \mathrm{~s}$ and two different signal-to-noise ratios (upper part) and two different signal-to-trend ratios (lower part)

\begin{tabular}{|c|c|c|c|c|c|}
\hline & $H=0.5$ & $H=0.6$ & $H=0.7$ & $H=0.8$ & $H=0.9$ \\
\hline & \multicolumn{5}{|c|}{ Signal-to-noise $=0$} \\
\hline MFDFA $_{\text {emd }}$ & 0.04 & 0.04 & 0.04 & 0.04 & 0.04 \\
\hline \multirow[t]{2}{*}{ GMWP } & 0.01 & 0.01 & 0.01 & 0.02 & 0.02 \\
\hline & \multicolumn{5}{|c|}{ Signal-to-noise $=0.33$} \\
\hline MFDFA $_{\text {emd }}$ & 0.03 & 0.04 & 0.04 & 0.05 & 0.06 \\
\hline \multirow[t]{2}{*}{ GMWP } & 0.02 & 0.02 & 0.03 & 0.04 & 0.05 \\
\hline & \multicolumn{5}{|c|}{ Signal-to-noise $=1$} \\
\hline MFDFA $_{\text {emd }}$ & 0.06 & 0.09 & 0.13 & 0.16 & 0.19 \\
\hline \multirow[t]{2}{*}{ GMWP } & 0.06 & 0.03 & 0.01 & 0.01 & 0.03 \\
\hline & \multicolumn{5}{|c|}{ Signal-to-trend $=0.33$} \\
\hline MFDFA $_{\text {emd }}$ & 0.29 & 0.22 & 0.16 & 0.10 & 0.07 \\
\hline \multirow[t]{2}{*}{ GMWP } & 0.34 & 0.24 & 0.14 & 0.05 & 0.02 \\
\hline & \multicolumn{5}{|c|}{ Signal-to-trend $=1$} \\
\hline MFDFA $_{\text {emd }}$ & 0.44 & 0.36 & 0.29 & 0.20 & 0.12 \\
\hline GMWP & 0.41 & 0.31 & 0.22 & 0.15 & 0.10 \\
\hline
\end{tabular}

analyses for multiharmonic trends with the larger signal-totrend ratio of 1 . In this case, the MFDFA, LWT, and entropy analyses underestimated the multifractal spectrum width, especially for the larger spectra indicated by a larger estimation error for $h_{\max }-h_{\min }=0.5$ and 0.6 in the lower part of Table 6. This increased estimation error was caused by a truncation of the right side (i.e., negative $q$ s) of the multifractal spectra (see red spectra in Fig. 7). MFDFA emd $_{\text {had an improved }}$ performance, as compared with the other analyses based on $q$ order statistics, for $H=0.5$ (see red trace in Fig. 7), but this result was not consistent across other $H$ s. In contrast, GMWP and $\mathrm{DFA}_{\text {loc }}$ were quite robust to the presence of large multiharmonic trends across $H \mathrm{~s}$ because of a narrow scaling range close to the limit $s \rightarrow 0$ (see the scaling range in Table 1).
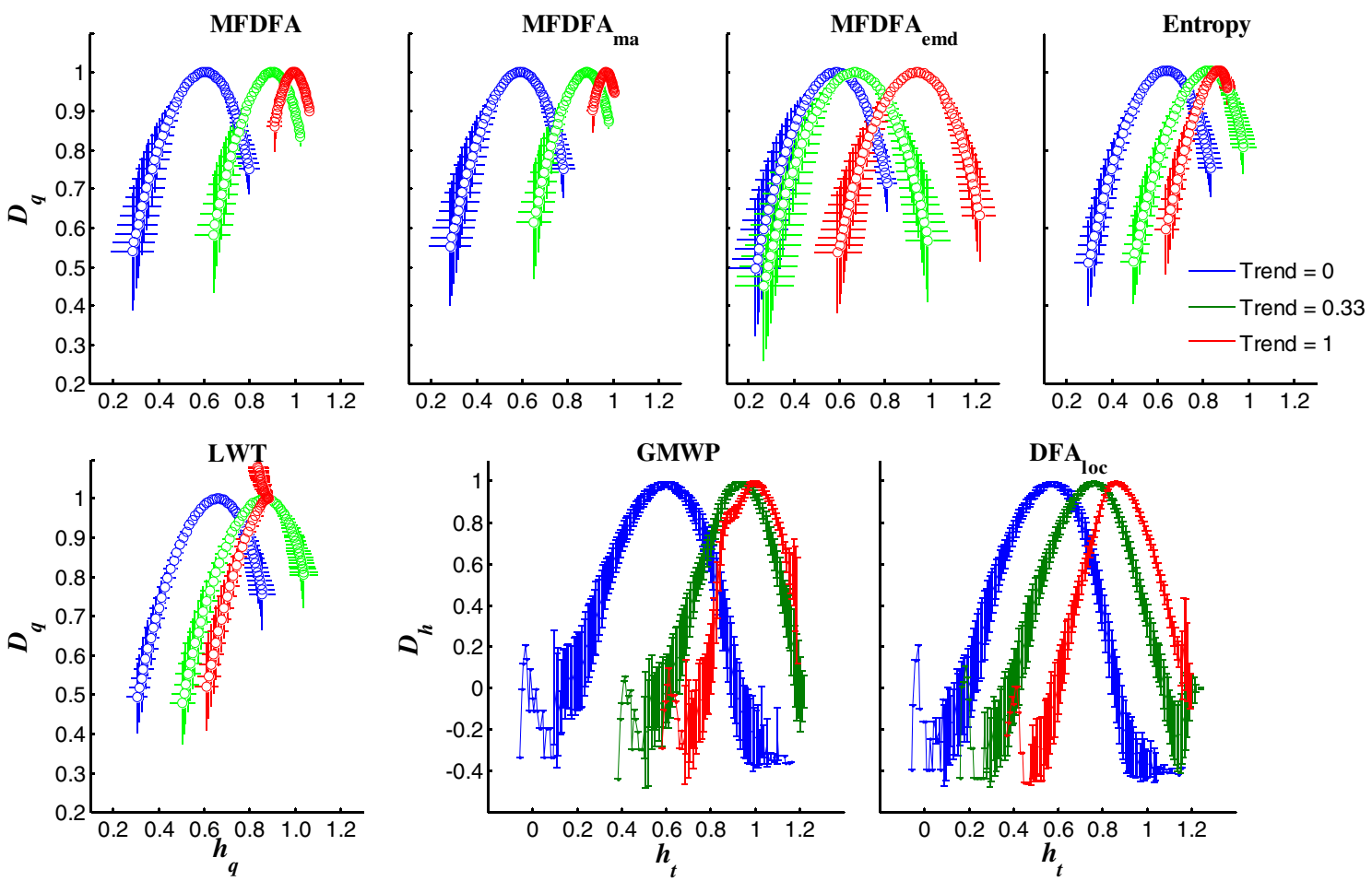

Fig. 7 Mean $D_{h}$ and $\pm 1 S D$ (bars) estimated by the seven multifractal analyses in Table 1 for the ensemble of $\Delta B_{H}(A(t))$ with multiharmonic trends. The estimated $D_{h}$ represented for no multiharmonic trend (blue

bars), a signal-to-trend ratio of 0.33 (green bars), and a signal-to-trend ratio of 1 (red bars). The parameter settings for $\Delta B_{H}(A(t))$ are $h_{\max }-h_{\min }$ $=0.6$ and $H=0.9$, and the sample size is 4,096 
Table 8 Mean error $E_{D}$ for six different multifractal spectrum widths $h_{\max }-h_{\min }$ for $\Delta B_{H}(A(t))$ with $H=0.5$ and with signal-to-trend ratio $=0.33$, but with no noise present

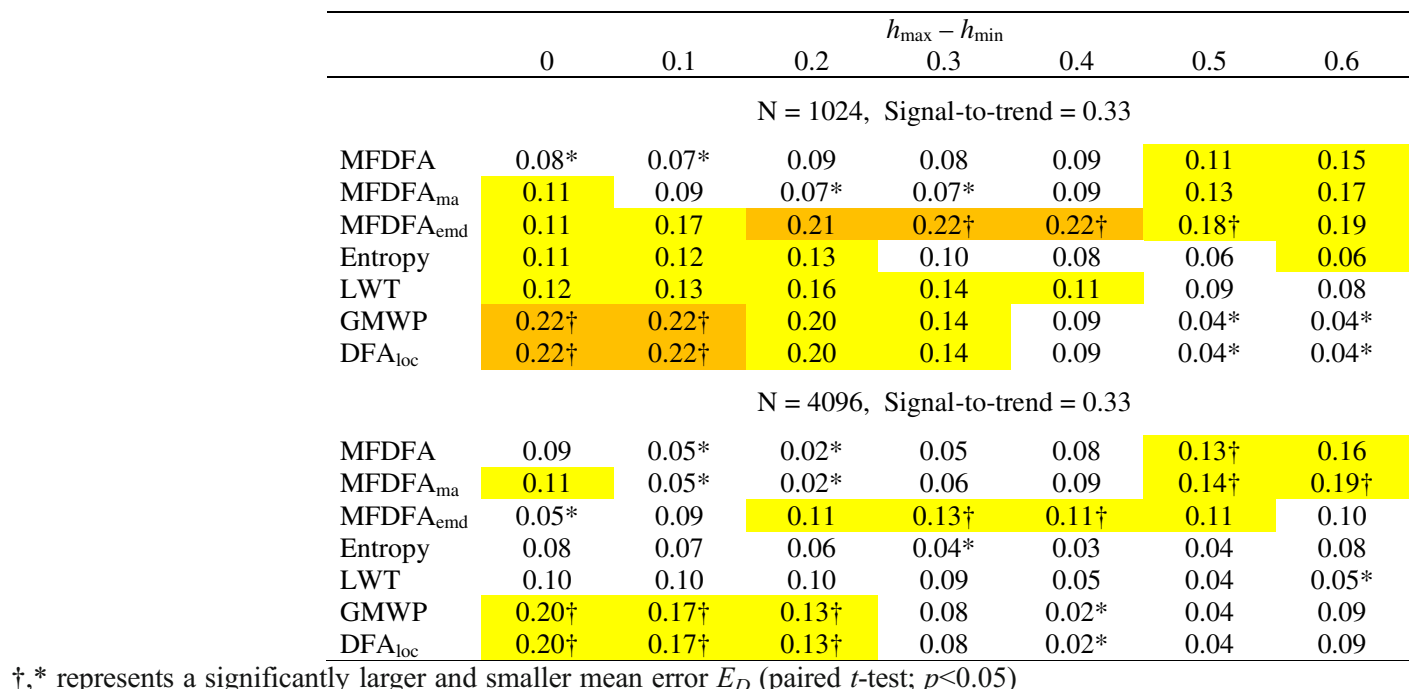

The central tendencies of the multifractal spectra were highly sensitive to the presence of multiharmonic trends with the low and high signal-to-trend ratios. The central tendency $\bar{h}$ for all $H$ of $\Delta B_{H}(A(t))$ was estimated close to $\bar{h}=1$, as indicated by large estimation errors $E_{\bar{h}}$ in the lower part of Table 7 for $H=0.5$ and 0.6 (see also the top of the red spectra in Fig. 7). Thus, multifractal analyses were not able to detect differences in the central tendency $\bar{h}$ when strong multiharmonic trends were present. Furthermore, the detrending procedures of both MFDFAs and wavelet-based analyses were not able remove the influence of the multiharmonic trends. As an example, MFDFA $_{\text {emd }}$, which contains the most adaptive detrending procedure, performed less precisely and less consistently than MFDFA with other detrending procedures when multiharmonic trends were present for the small sample size of $N=1,024$ (see crucifix in the upper part of Table 8 and lower part of Table 7).

\section{Discussion}

The purpose of the present study was to compare the performance of seven multifractal analyses to provide a guide for the choice of multifractal analyses of response time series and other behavioral variables. In the discussion, the present comparative study of multifractal analyses is compared with the results in previous comparative studies. In addition, the implications of the sensitivity of the multifractal analyses to additive noise and multiharmonic trends are discussed in the last subsection.

\section{MFDFA $_{\text {lin }}$ versus MFDFA $_{\text {ma }}$ and MFDFA $_{\text {emd }}$}

The performance of MFDFA was found in previous studies to be dependent on the detrending procedures. MFDFA and MFDFA $_{\text {emd }}$ have been shown to outperform the original MFDFA $_{\text {lin }}$ when employed to a simple binominal cascade (Gu \& Zhou, 2010; Manimaran, Panigrahi, \& Parikh, 2009; Qian, Zhou, \& Gu, 2009). In the present study, MFDFA and MFDFA $_{\text {emd }}$ had similar or larger estimation errors, as compared with the original MFDFA ${ }_{\text {lin }}$. The deviation of the performance of the detrending procedures in the present study, as compared with previous studies, is probably caused by smaller sample sizes and a more advanced multiplicative cascading model used in the present study.

The poor performance of the detrending procedures of the MFDFA and wavelet-based analyses indicates that the detrending procedure has to be exactly matched to the shape of a complex multiharmonic trend in order for these procedures to remove the influence of trends on the estimated $D_{h}$. The exact tuning of detrending procedures will be extremely difficult in multifractal analyses of response time series and other behavioral variables where a priori theoretical argumentations for a particular shape of a complex trend do not exist. Thus, the comparison of the performances of the original MFDFA $\mathrm{lin}_{\text {lin }}$ versus MFDFA ${ }_{\text {ma }}$ and MFDFA $_{\text {emd }}$ suggests that the original MFDFA ${ }_{\text {lin }}$ should be used unless theoretical argumentation exists for the exact shape of the trend.

\section{LWT versus MFDFA}

Serrano and Figliola (2009) concluded that LWT gave an improved estimation of $D_{h}$, as compared with MFDFA, when employed to binominal and random wavelet cascades. In contrast, X. Y. Huang et al. (2011) found that MFDFA performed better than LWT considering only positive $q$ s when employed on canonical Mandelbrot cascades. The present study confirms the findings by X. Y. Huang et al. that MFDFA had better 
performance than LWT for $\Delta B_{H}(A(t))$ with $H=0.5$. However, LWT had a more consistent error $E_{D}$ than the MFDFA and entropy analyses across the range of $H \mathrm{~s}$ of $\Delta B_{H}(A(t))$ and had significantly better performance for $H=0.8$ and 0.9 . Furthermore, LWT seems to have a more consistent performance, as compared with both the MFDFA and entropy analyses, when estimating the left side (i.e., positive $q \mathrm{~s}$ ) of the multifractal spectrum $D_{h}$. Thus, LWT is a good choice of multifractal analyses of response time series, especially for long sample sizes of 4,096 and when positive $q$ s are considered.

\section{Entropy analysis versus MFDFA and LWT}

Entropy analysis has recently been suggested as a superior estimation procedure of $D_{h}$ of response time series, as compared with the multifractal analyses based on the Legendre transformation in Eq. 6 (Stephen \& Dixon, 2011). This suggestion is based on the original argumentation by Chhabra and Jensen (1989) in their development of entropy analysis. However, Chhabra and Jensen compared entropy analysis with a basic histogram analysis based on Eqs. 8 and 9. The present study shows only minor differences between the entropy analysis of Chhabra and Jensen and MFDFA and LWT. Furthermore, the probability measure used in entropy analysis (i.e., Eq. 2) can be constructed by dividing other choices of scale-dependent measure $\mu_{s, v}$ by the total sum of $\mu_{s, t_{0}}$. Substituting the original scale-dependent measure in entropy analysis (i.e., Eq. 7a) with these probability measures will yield the exact same $D_{h}$ as the Legendre transformation of $\zeta_{q}$ in Eq. 6 . Thus, the performance of multifractal analyses with similar $\mu_{s, v}$ is insensitive to the choice of estimating $D_{h}$ directly by entropy functions or estimating $D_{h}$ indirectly by the Legendre transformation of $\zeta_{q}$.

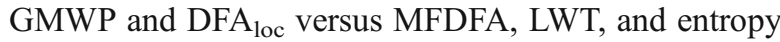
analysis

The benchmark test performed by Turiel et al. (2008) concluded superior performance by GMWP, especially for multifractal signals with large spectrum width $h_{\max }-h_{\min }$ and small sample sizes (i.e., $N=1,024)$. The present study also found that both GMWP and $\mathrm{DFA}_{\text {loc }}$ performed better than the multifractal analyses based on $q$-order statistics (i.e., MFDFA, LWT, and entropy analysis) for the larger spectrum width. However, GMWP and DFA $A_{\text {loc }}$ both had a larger overestimation of the spectrum width of monofractal signals, as compared with MFDFA, LWT, and entropy analysis. A shortcoming of the benchmark test conducted by Turiel et al. (2008) was the inclusion of other multifractal analyses based on $q$-order statistics, which have inferior performance, as compared with analyses included in the present study (Jaffard, Lashermes, \& Abry, 2006; Oświęcimka, Kwapien, \& Drozdz, 2006; Serrano \&
Figliola, 2009). Another shortcoming of the benchmark test conducted by Turiel et al. (2008) was the use of random cascades that are not appropriate candidates to mimic the intermittent variation in behavioral variables by their scale-discrete nature and strictly positive values. Furthermore, Pont, Turiel, and Perez-Vicente (2006) have shown that the GMWP overestimates the spectrum widths for fractional Gaussian noise (i.e., $\Delta B_{H}(A(t))$ with $h_{\max }-h_{\min }=0$ ) confirmed in the present result. However, the same authors showed that the performance of both GMWP and $\mathrm{DFA}_{\mathrm{loc}}$ can be improved by modifications of the scale-dependent measure $\mu_{s, t_{0}}$ when employed to monofractal $\Delta B_{H}(A(t))$. In summary, the present study is less conclusive regarding the performance of both GMWP and $\mathrm{DFA}_{\text {loc }}$, as compared with the benchmark test conducted by Turiel et al. (2008). The obtained $D_{h}$ from GMWP and $\mathrm{DFA}_{\mathrm{loc}}$ of response time series should be compared with $D_{h}$ of monofractal surrogate series to prevent false positive presence of multifractality.

Sensitivity to additive noise and multiharmonic trends

The presence of both trends and noise yields a false negative result for the presence of multifractal response time variation, a false positive result for the presence of long-range trial dependency when trends dominate, and a false negative result for long-range trial dependency when noise dominates. The sensitivity of multifractal analyses to noise and trends has two important implications for the employment of multifractal analyses to response time series and the interpretation of the estimated $D_{h}$.

First, a narrow $D_{h}$ reflects the lack of a multiplicative interaction or phase coupling between temporal scales of response time series when no noise or trends are present. Ihlen and Vereijken (2010) reported multifractal spectra with smaller widths and central tendencies closer to 1 for the response series of the interval estimation task, as compared with other cognitive tasks. Furthermore, Kuznetsov and Wallot (2011) reported that the local singularity exponent $h_{t}$ of a response series in an interval estimation task had less variation (i.e., smaller spectrum width) and an average value (i.e., central tendency) closer to 0.5 in the presence of performance feedback. The sensitivity of multifractal analyses to noise and trends suggests that both these results can be created by task- and feedback-dependent modulation of additive noise and trends. Thus, noise and trends will disguise the task and feedback modulation of multifractal spectra caused by modulation of the intrinsic multiplicative interactions between temporal scales. However, both the Ihlen and Vereijken (2010) and Kuznetsov and Wallot studies found significant differences between the multifractal spectrum of the response time series and multifractal spectra of synthesis surrogate series that eliminate multiplicative interactions between the temporal scales within the response time series. Furthermore, Kuznetsov and Wallot found no correlation between the 
Table 9 Summary of all pros and cons of the seven multifractal analyses in Table 1

\begin{tabular}{|c|c|c|}
\hline & Pros & Cons \\
\hline & Estimation of $D_{h}$ indirectly from the $q$-order statistics of $\mu_{s, v}$ & \\
\hline MFDFA & $\begin{array}{l}\text { - Superior performance for } H \rightarrow 0.5 \text { for small sample size } \\
\text { - Polynomial detrending }\end{array}$ & $\begin{array}{l}\text { - Inferior performance when } H \rightarrow 1 \text { for small sample size } \\
\text { - Inconsistent performance across } H \text { for small sample size } \\
\text { - Trend and noise sensitive }\end{array}$ \\
\hline MFDFA $_{\text {ma }}$ & $\begin{array}{l}\text { - Superior performance for } H \rightarrow 0.5 \text { for small sample size } \\
\text { - Moving average detrending }\end{array}$ & $\begin{array}{l}\text { - Inferior performance when } H \rightarrow 1 \text { for small sample size } \\
\text { - Inconsistent performance across } H \text { for small sample size } \\
\text { - Trend and noise sensitive }\end{array}$ \\
\hline MFDFA $_{\text {emd }}$ & $\begin{array}{l}\text { - Consistent performance with the other MFDFA for large sample size } \\
\text { - Detrending by empirical mode decomposition } \\
\text { - Superior detrending for } H \rightarrow 0.5\end{array}$ & $\begin{array}{l}\text { - Inferior performance for small sample size } \\
\text { - Inconsistent performance across } H \text { for small sample size } \\
\text { - Trend and noise sensitive }\end{array}$ \\
\hline Entropy & $\begin{array}{l}\text { - Superior performance for large sample size } \\
\text { - Consistent performance across } H\end{array}$ & $\begin{array}{l}\text { - Inferior performance for } H \rightarrow 0.5 \text { for small sample size } \\
\text { - No detrending } \\
\text { - Trend and noise sensitive }\end{array}$ \\
\hline LWT & $\begin{array}{l}\text { - Superior performance for large sample size } \\
\text { - Consistent performance across } H \\
\text { - More consistent estimation error for positive } q \text { 's } \\
\text { - Wavelet detrending }\end{array}$ & $\begin{array}{l}\text { - Inferior performance for } H \rightarrow 0.5 \text { for small sample size } \\
\text { - Trend and noise sensitive } \\
\text { - Inferior estimation of the central tendency of } D_{h}\end{array}$ \\
\hline & Estimation of $D_{h}$ directly from $\mu_{s, t_{0}}$ & \\
\hline GMWP & $\begin{array}{l}\text { - Superior estimation of } D_{h} \text { with large widths } \\
\text { - Superior estimation of the central tendency of } D_{h} \\
\text { - Wavelet detrending } \\
\text { - Relatively trend resistant }\end{array}$ & $\begin{array}{l}\text { - Overestimation of } D_{h} \text { widths for monofractal signals } \\
\text { - Inconsistent performance across } H \text { for small sample size } \\
\text { - Less consistent estimation error of } D_{h} \text { and central tendency of } D_{h} \\
\text { - Noise sensitive }\end{array}$ \\
\hline $\mathrm{DFA}_{\mathrm{loc}}$ & $\begin{array}{l}\text { - Superior estimation of } D_{h} \text { with large widths } \\
\text { - Consistent estimation error for large sample size } \\
\text { - More consistent estimation error of central tendency of } D_{h} \\
\text { - Polynomial detrending } \\
\text { - Relatively trend resistant }\end{array}$ & $\begin{array}{l}\text { - Overestimation of } D_{h} \text { widths for monofractal signals } \\
\text { - Inconsistent performance across } H \text { for small sample size } \\
\text { - Noise sensitive }\end{array}$ \\
\hline
\end{tabular}

multifractal spectrum width and the central tendency of the spectrum that would have been expected in the presence of a high level of noise or strong trends. In summary, surrogate tests and correlation analyses of multifractal spectrum widths and the central tendencies should be conducted together with the multifractal analyses to control for the presence of additive noise and trends.

Second, the presence of noise and trends leads to a degeneration of $D_{h}$ and deviation from linearity in the log-scaling plot of the $q$-order statistics for the multifractal analyses based on the $q$-order statistics. The degeneration of $D_{h}$ appeared in the right end of $D_{h}$ and was caused by a nonmonotonic decreasing $h_{q}$ for negative $q$ s (see red trace estimated by LWT in Fig. 7). The deviation from linearity in the log-scaling plot was also most apparent for negative $q$ s and suggests that noise and trends with large magnitudes will influence the scale invariance of response time series, especially for the intermittent periods with response time variation of small magnitudes. Consequently, the employment of multifractal analyses based on $q$-order statistics should interpret the right end of the estimated $D_{h}$ with caution when noise and trends are expected.

\section{Conclusion}

Table 9 summarizes the pros and cons of the seven multifractal analyses compared in the present study. All multifractal analyses were able to differentiate between 
monofractal and multifractal time series, but GMWP and $D^{2} A_{l o c}$ showed a larger overestimation of the multifractal spectrum size for the monofractal time series. However, GMWP and $\mathrm{DFA}_{\text {loc }}$ were more robust to multiharmonic trends of large magnitudes, and GMWP had the most precise estimation of the central tendency of the multifractal spectrum. MFDFA $A_{\text {lin }}$ and MFDFA ${ }_{\text {ma }}$ had a superior performance for $H=0.5$, as compared with both LWT and entropy analysis. However, the LWT and entropy analyses had more consistent performances across $H$ and had superior performances, as compared with MFDFA, for $H=0.9$. The detrending procedures of MFDFA and the wavelet-based analyses were unable to remove the influence of a multiharmonic trend on the estimated multifractal spectra. The present study concludes that the estimated multifractal spectrum from two or more multifractal analyses should be compared with response time series and other behavioral data when these analyses are employed. In addition, the estimated multifractal spectra should be compared with the estimated spectra from monofractal surrogate series to reveal a false positive presence of multifractality.

Acknowledgement The multiplicative cascading noise $\Delta B_{H}(A(t))$ are synthesized by MATLAB functions available at http://www.isima. $\mathrm{fr} / \sim$ pchainai/PUB/software.html. The EMD is available at http:// perso.ens-lyon.fr/patrick.flandrin/software $2 . \mathrm{html}$. The MATLAB codes of the multifractal analyses in Table 1 are available at www.ntnu.edu/inm/geri/ software. The MATLAB codes for LWT are developed by Wendt (2008) and are available at http://perso.ens-lyon.fr/herwig.wendt/index.php?page= codes.

\section{Appendix 1 Detrending procedures in MFDFA}

Linear detrending $\left(\mathrm{MFDFA}_{\text {lin }}\right)$

The scale-dependent measure $\mu_{s, v}$ of the original MFDFA $\mathrm{Iin}_{\text {in }}$ is defined by the following equation (Kantelhardt et al., 2002):

$$
\mu_{s, v}=\sqrt{\frac{1}{s} \sum_{t=(v-1) s+1}^{v s}\left[y_{t}-\hat{y}_{t, m}\right]^{2}}
$$

The integrated profile $y_{t}=\sum_{i=1}^{t}\left(x_{i}-\bar{x}\right)$ of the response time series $x_{i}$ is divided into $N_{s}=N / s$ nonoverlapping segments with segment number $v=1, \ldots, N_{s}$ where each segment contains the response times of $s$ trials. The polynomial trend of order $m$ is least square fitted to $y_{t}$ within each segment $v$ and was chosen to be $m=1$ (i.e., linear) in the present study.

Moving average detrending (MFDFA ${ }_{\text {ma }}$ )

An alternative way to provide a scale-dependent detrending in the computation of $\mu_{s, v}$ is to use a moving average detrending procedure (Gu \& Zhou, 2010):

$$
\mu_{s, v}=\sqrt{\frac{1}{s} \sum_{t=(v-1) s+1}^{v s}\left[y_{t}-\widehat{y}_{s, t}\right]^{2}}
$$

where the scale-dependent trend $\hat{y}_{s, t}$ is the moving average of $y_{t}$ with a floating trial interval $[t-s / 2, t+s / 2]$ that contains the response time of $s$ trials:

$$
\widehat{y}_{s, t}=\frac{1}{s} \sum_{i=t-s / 2}^{t+s / 2} y_{i}
$$

Empirical mode decomposition detrending $\left(\mathrm{MFDFA}_{\mathrm{emd}}\right)$

The scale-dependent trend $\widehat{y}_{s, t}$ in Eq. 13 can also be defined by the intrinsic mode functions $d_{s, t}$ defined by empirical mode decomposition (EMD) of $y_{t}$ (N. E. Huang et al., 1998):

$$
\widehat{y}_{s, t}=\sum_{\lceil s\rceil} d_{s, t}
$$

where $\lceil s\rceil$ indicates that the sum is taken over all intrinsic mode functions $d_{s, t}$ with a scale larger than $s$. The scale of each $d_{s, t}$ was defined by the inverse of their mean instant frequency $f_{s, t}$ (i.e., $s=1 /\left\langle f_{s, t}\right\rangle_{t}$ ), where $f_{s, t}$ was computed by differentiating the phase angle of the Hilbert transform of $d_{s, t}$ (i.e. $f_{s, t}=d \theta_{s, t} / d t$ ) by an algorithm suggested by Boashash (1992). It is important to notify that this detrending procedure defines an EMD modification of the MFDFA suggested by Manimiran et al. (2009) and is, therefore, different from the EMD modification of MFDFA developed by Qian et al. (2009). MATLAB codes for $\mathrm{MFDFA}_{\text {lin }}$ and $\mathrm{MFDFA}_{\text {emd }}$ are available at www.ntnu.edu/inm/geri/software, whereas the MATLAB code for $\mathrm{MFDFA}_{\mathrm{ma}}$ is available in the Appendix in $\mathrm{Gu}$ and Zhou (2010). 


\section{Appendix 2 Estimation of the local singularity exponent $\boldsymbol{h}_{t}$}

The local singularity exponent was estimated by a method developed by Struzik (2000). The method assumes that the time series is generated by a multiplicative cascading process where the temporal variation of the scale-dependent measure $\mu_{s, t_{0}}$ decays with increasing scale $s$. The local singularity exponent $h_{t_{0}}$ in Eq. 8 is estimated by the coarse-grained singularity exponents $h_{s, t_{0}}$ in the limit $s \rightarrow 0$. The coarsegrained singularity exponent is defined by the following equation (Ihlen, 2012; Struzik, 2000):

$h_{s, t_{0}}=\frac{\log \left(\mu_{s, t_{0}}\right)-(\bar{h} \log (s)+C)}{\log (s)-\log \left(s_{\max }\right)}+\bar{h} \quad s \rightarrow 0$

$\bar{h} \log (s)+C$ is the regression line at scale $s$ with the slope $\bar{h}$ and estimated by a $q$-order based analysis for $q=0$. The scale-dependent measure $\mu_{s, t_{0}}$ was defined by Eq. 3 for GMWP and by Eq. 4 for $\mathrm{DFA}_{\text {loc. }}$. The maximum scale $s_{\max }$ was set to the length of the time series (Struzik, 2000). The MATLAB codes for both GMWP and DFA $\mathrm{Acc}_{\text {loc }}$ are available at www.ntnu.edu/inm/geri/software.

\section{References}

Bacry, E., Muzy, J. F., \& Delour, J. (2001). Multifractal random walk. Physical Review E, 64, 026103-026106.

Boashash, B. (1992). Estimating and interpretating the instantaneous frequency of a signal part 2: algorithms and applications. Proceedings of the IEEE, 80, 540-568.

Burbeck, S. L., \& Luce, R. D. (1982). Evidence from auditory simple reaction times for both change and level detectors. Perception \& Psychophysics, 32, 117-133.

Canus, C., Lévy Véhel, J., \& Tricot, C. (1998). Continuous large deviation multifractal spectrum: definition and estimation. In Fractals 98, Malta.

Chainais, P., Riedi, R., \& Abry, P. (2005). On non-scale-invariant infinitely divisible cascades. IEEE Transaction on Information Theory, 51(3), 1063-1083.

Chhabra, A., \& Jensen, R. V. (1989). Direct determination of the $\mathrm{f}(\alpha)$ singularity Spectrum. Physical Review Letters, 62, 1327-1330.

Daubechies, I. (1992). Ten lectures on wavelets. Philadelphia, PA: SIAM.

Delignières, D., Ramdani, S., Lemoine, L., Torre, K., Fortes, M., \& Ninot, G. (2006). Fractal analysis for short time series: a reassessment of classical methods. Journal of Mathematical Psychology, 50, 525-544.

Diniz, A., Wijnants, M. L., Torre, K., Barreiros, J., Crato, N., Bosman, A. M. T., et al. (2011). Contemporary theories of $1 / f$ noise in motor control. Human Movement Science, 30(5), 889-905.

Gilden, D. L. (2001). Cognitive emissions of $1 / f$-noise. Psychological Review, 108(1), 33-56.

Gilden, D. L., Thornton, T., \& Mallon, M. W. (1995). 1/f-noise in human cognition. Science, 267, 1837-1839.

Gu, G.-F., \& Zhou, W.-X. (2010). Detrending moving average algorithm for multifractals. Physical Review E, 82, 011136.
Holden, J. G., Van Orden, G. C., \& Turvey, M. T. (2009). Dispersion of response times reveals cognitive dynamics. Psychological Review, $116(2), 318-342$.

Huang, X. Y., Schmitt, F. G., Hermand, J.-P., Gagne, Y., Lu, Z. M., \& Liu, Y. L. (2011). Arbitrary-order Hilbert spectral analysis for time series possessing scaling statistics: a comparison study with detrended uctuation analysis and wavelet leaders. Physical Review E, 84, 016208.

Huang, N. E., Shen, Z., Long, S. R., Wu, M. C., Shih, H. H., Zheng, Q., et al. (1998). The empirical mode decomposition and the Hilbert spectrum for nonlinear and non-stationary time series analysis. Proceedings of the Royal Society A, 454, 903-995.

Ihlen, E. A. F. (2012). Introduction to multifractal detrended fluctuation analysis in Matlab. Frontiers in Physiology: Fractal Physiology, 3(141), 1-18.

Ihlen, E. A. F., \& Vereijken, B. (2010). Interaction dominant dynamics in human cognition: beyond $1 / f^{\alpha}$ fluctuations. Journal of Experimental Psychology. General, 139, 436-463.

Ivanov, P. C., Rosenblum, M. G., Amaral, L. A. N., Struzik, Z. R., Havlin, S., Goldberger, A. L., et al. (1999). Multifractality in human heartbeat dynamics. Nature, 399, 461-465.

Jaffard, S., Lashermes, B., \& Abry, P. (2006). Wavelet leaders in multifractal analysis. In T. Qian, M. I. Vai, \& Y. Xu (Eds.), Wavelet analysis and applications (pp. 219-264). Basel: Birkhäuser Verlag.

Kantelhardt, J. W., Zschiegner, S. A., Koscielny-Bunde, E., Havlin, S., Bunde, A., \& Stanley, H. E. (2002). Multifractal detrended fluctuation analysis of nonstationary time series. Physica A, 316, 87114.

Kello, C. T., Beltz, B. C., Holden, J. G., \& Van Orden, G. C. (2007). The emergent coordination of cognitive function. Journal of Experimental Psychology. General, 136, 551-568.

Kello, C. T., Brown, G. D. A., Ferrer-i-Cancho, R., Holden, J. G., Linkenkaer-Hansen, K., Rhodes, T., et al. (2010). Scaling laws in cognitive sciences. Cell Press: Trends in Cognitive Sciences, 14(5), 223-232.

Kelso, J. A. S. (1995). Dynamic pattern: The self-organization of brain and behavior. Cambridge: Mass, MIT Press.

Kuznetsov, N. A., \& Wallot, S. (2011). Effects of accuracy feedback on fractal characteristics of time estimation. Frontiers in Integrative Neuroscience, 5(62), 1-12.

Logan, G. D. (1992). Shapes of reaction-time distributions and shapes of learning curves: a test of the instance theory of automaticity. Journal of Experimental Psychology: Learning, Memory, and Cognition, 18, 883-914.

Luce, R. D. (1986). Response times: Their role in inferring elementary mental organization. Oxford: Oxford University Press.

Mallat, S. (1999). A wavelet tour in signal processing (2nd ed.). San Diego: Academic Press.

Manimaran, P., Panigrahi, P. K., \& Parikh, J. C. (2009). Multiresolution analysis of fluctuations in non-stationary time series through discrete wavelets. Physica A, 388, 2306-2314.

Moscoso del Prado Martin, F. (2009). Scale-invariance of human latencies. In N. Taatgen \& H. Van Rijn (Eds.), Proceedings of the 31st annual conference of the cognitive science society (pp. 1270-1275). Austin: Cognitive Science Society.

Oświęcimka, P., Kwapien, J., \& Drozdz, S. (2006). Wavelet versus detrended fluctuation analysis of multifractal structures. Physical Review E, 74(1), 016103.

Parisi, G., \& Frisch, U. (1985). On the singularity structure of fully developed turbulence. In M. Ghil, R. Benzi, \& G. Parisi (Eds.), Turbulence and predictability in geophysical fluid dynamics, proceedings of the international school of physics (pp. 84-87). North Holland: Amsterdam. 
Pont, O., Turiel, A., \& Perez-Vicente, C. J. (2006). Application of microcanonical multifractal formalism to monofractal systems. Physical Review E, 74, 061110.

Qian, X.-Y., Zhou, W.-X., \& Gu, G.-F. (2009). Modified detrended fluctuation analysis based on empirical mode decomposition. http://arxiv.org/PS_cache/arxiv/pdf/0907/0907.3284v1.pdf

Schaffer, M., Bascompte, J., Brock, W. A., Brovkin, V., Carpenter, S. R., Dakos, V., et al. (2009). Early-warning signals for critical transitions. Nature, 461, 53-59.

Schwarz, W. (2001). The ex-Wald distribution as a descriptive model of response times. Behavior Research Methods, Instruments, \& Computers, 33(4), 457-469.

Serrano, E., \& Figliola, A. (2009). Wavelet leaders: a new method to estimate the multifractal singularity spectra. Physica A, 388, 2793-2805.

Stephen, D. G., \& Dixon, J. A. (2011). Strong anticipation: multifractal cascade dynamics modulate scaling in synchronization behaviors. Chaos, Solitions and Fractals, 44, 160-168.

Struzik, Z. R. (2000). Determining local singularity strength and their spectra with the wavelet transform. Fractals, 8(2), 163-179.

Turiel, A., \& Perez-Vicente, C. J. (2003). Multifractal geometry in stock market time series. Physica A, 322, 629-649.

Turiel, A., Perez-Vicente, C. J., \& Grazzini, J. (2006). Numerical methods for the estimation of the estimation of the multifractal singularity spectra on sampled data: a comparative study. Journal of Computational Physics, 216, 362-390.

Turiel, A., Yahia, H., \& Pérez-Vicente, C. J. (2008) Microcanonical multifractal formalism: a geometrical approach to multifractal systems. Part I: singularity analysis. Journal of Physics A: Mathematical and General, 41, 015501.

Ulrich, R., \& Miller, J. (1993). Information processing models generating lognormally distributed reaction times. Journal of Mathematical Psychology, 37, 513-525.

Van Orden, G. C., Holden, J. G., \& Turvey, M. T. (2003). Selforganization of cognitive performance. Journal of Experimental Psychology. General, 132(3), 331-350.

Van Zandt, T., \& Ratcliff, R. (1995). Statistical mimicking of reaction time data: Single process models, parameter variability, and mixtures. Psychonomic Bulletin and Review, 2, 20 54.

Wang, G., Huang, H., Xie, H., Wang, Z., \& Hu, X. (2007). Multifractal analysis of ventricular fibrillation and ventricular tachycardia. Medical Engineering \& Physics, 29(3), 375-379.

Wendt, H. (2008). Contribution of wavelet leaders and bootstrap to multifractal analysis: Images, Estimation performance, Dependence structure and vanishing moments, Confidence intervals and Hypothesis tests. $\mathrm{PhD}$ thesis, Ecole Normale Superieure de Lyon, Lyon. 